\title{
Well-Posedness in Smooth Function Spaces for Moving-Boundary 1-D Compressible Euler Equations in Physical Vacuum
}

\author{
DANIEL COUTAND \\ Heriot-Watt University \\ STEVE SHKOLLER \\ University of California, Davis
}

\begin{abstract}
The free-boundary compressible one-dimensional Euler equations with moving physical vacuum boundary are a system of hyperbolic conservation laws that are both characteristic and degenerate. The physical vacuum singularity (or rate of degeneracy) requires the sound speed $c^{2}=\gamma \rho^{\gamma-1}$ to scale as the square root of the distance to the vacuum boundary and has attracted a great deal of attention in recent years. We establish the existence of unique solutions to this system on a short time interval, which are smooth (in Sobolev spaces) all the way to the moving boundary. The proof is founded on a new higher-order, Hardy-type inequality in conjunction with an approximation of the Euler equations consisting of a particular degenerate parabolic regularization. Our regular solutions can be viewed as degenerate viscosity solutions. (C) 2010 Wiley Periodicals, Inc.
\end{abstract}

\section{Introduction}

\subsection{Compressible Euler Equations and the Physical Vacuum Boundary}

This paper is concerned with the evolving vacuum state in a compressible gas flow. For $0 \leq t \leq T$, the evolution of a one-dimensional compressible gas moving inside of a dynamic vacuum boundary is modeled by the one-phase compressible Euler equations:

$$
\begin{aligned}
\rho\left[u_{t}+u u_{x}\right]+p(\rho)_{x} & =0 & & \text { in } I(t), \\
\rho_{t}+(\rho u)_{x} & =0 & & \text { in } I(t), \\
p & =0 & & \text { on } \Gamma(t), \\
\mathcal{V}(\Gamma(t)) & =u, & & \\
(\rho, u) & =\left(\rho_{0}, u_{0}\right) & & \text { on } I(0), \\
I(0) & =I=\{0<x<1\} . & &
\end{aligned}
$$

The open, bounded interval $I(t) \subset \mathbb{R}$ denotes the changing domain occupied by the gas, $\Gamma(t):=\partial I(t)$ denotes the moving vacuum boundary, and $\mathcal{V}(\Gamma(t))$ denotes the 
velocity of $\Gamma(t)$. The scalar field $u$ denotes the Eulerian velocity field, $p$ denotes the pressure function, and $\rho$ denotes the density of the gas. The equation of state $p(\rho)$ is given by

$$
p(x, t)=C_{\gamma} \rho(x, t)^{\gamma} \quad \text { for } \gamma>1 \text {, }
$$

where $C_{\gamma}$ is the adiabatic constant, which we set to 1 , and

$$
\rho>0 \text { in } I(t) \text { and } \rho=0 \text { on } \Gamma(t) .
$$

Equation (1.1a) is the conservation of momentum, (1.1b) is the conservation of mass, the boundary condition (1.1c) states that the pressure (and hence density) must vanish along the vacuum boundary, (1.1d) states that the vacuum boundary is moving with the fluid velocity, and (1.1e)-(1.1f) are the initial conditions for the density, velocity, and domain. Using the equation of state (1.2), (1.1a) is written as

$$
\rho\left[u_{t}+u u_{x}\right]+\left(\rho^{\gamma}\right)_{x}=0 \text { in } I(t) .
$$

With the sound speed given by $c^{2}(x, t)=\gamma \rho^{\gamma-1}(x, t)$, and with $c_{0}=c(x, 0)$, the condition

$$
0<\left|\frac{\partial c_{0}^{2}}{\partial x}\right|<\infty \quad \text { on } \Gamma
$$

defines a physical vacuum boundary (or singularity) (see $[10,12,13,17])$. Since $\rho_{0}>0$ in $I,(1.3)$ implies that for some positive constant $C$ and $x \in I$ near the vacuum boundary $\Gamma:=\partial I$,

$$
\rho_{0}^{\gamma-1}(x) \geq C \operatorname{dist}(x, \Gamma) .
$$

Equivalently, the physical vacuum condition (1.4) implies that for some $\alpha>0$,

$$
\left|\frac{\partial \rho_{0}^{\gamma-1}}{\partial x}(x)\right| \geq C_{\alpha}>0 \quad \text { for any } x \text { satisfying } d(x, \partial I) \leq \alpha,
$$

and for a constant $C_{\alpha}$, depending on $\alpha$,

$$
\rho_{0}^{\gamma-1}(x) \geq C_{\alpha}>0 \quad \text { for any } x \text { such that } d(x, \partial I) \geq \alpha .
$$

Because of condition (1.4), the compressible Euler system (1.1) is a degenerate and characteristic hyperbolic system to which standard methods of symmetric (uniformly) hyperbolic conservation laws cannot be applied in standard Sobolev spaces. In [1], we established a priori estimates for the multidimensional compressible Euler equations with physical vacuum boundary.

The main result of this paper is the construction of unique solutions in the onedimensional case, which are smooth all the way to the moving vacuum boundary on a (short) time interval $[0, T]$, where $T$ depends on the initial data. We combine the methodology of our a priori estimates [1], with a particular degenerate parabolic regularization of the Euler equations, which follows our methodology in [2, 3], as well as a new higher-order Hardy-type inequality that permits the construction of solutions to this degenerate parabolic regularization. As we describe below in 
Section 1.7, our solutions can be thought of as degenerate viscosity solutions. The multidimensional existence theory is treated in [4].

\subsection{Fixing the Domain and Lagrangian Variables on Reference Interval $I$}

We transform system (1.1) into Lagrangian variables. We let $\eta(x, t)$ denote the "position" of the gas particle $x$ at time $t$. Thus,

$$
\partial_{t} \eta=u \circ \eta \quad \text { for } t>0 \text { and } \eta(x, 0)=x
$$

where $\circ$ denotes composition so that $[u \circ \eta](x, t):=u(\eta(x, t), t)$. We set

$$
\begin{aligned}
& v=u \circ \eta(\text { Lagrangian velocity), } \\
& f=\rho \circ \eta(\text { Lagrangian density }) .
\end{aligned}
$$

The Lagrangian version of equations (1.1a)-(1.1b) can be written on the fixed reference domain $I$ as

$$
\begin{aligned}
f v_{t}+\left(f^{\gamma}\right)_{x} & =0 & & \text { in } I \times(0, T], \\
f_{t}+f v_{x} / \eta_{x} & =0 & & \text { in } I \times(0, T], \\
f & =0 & & \text { in } I \times(0, T], \\
(f, v, \eta) & =\left(\rho_{0}, u_{0}, e\right) & & \text { in } I \times\{t=0\},
\end{aligned}
$$

where $e(x)=x$ denotes the identity map on $I$.

It follows from solving equation (1.8b) that

$$
f=\rho \circ \eta=\frac{\rho_{0}}{\eta_{x}}
$$

so that the initial density function $\rho_{0}$ can be viewed as a parameter in the Euler equations. Let $\Gamma:=\partial I$ denote the initial vacuum boundary; we write the compressible Euler equations (1.8) as

$$
\begin{aligned}
\rho_{0} v_{t}+\left(\rho_{0}^{\gamma} / \eta_{x}^{\gamma}\right)_{x} & =0 & & \text { in } I \times(0, T], \\
(\eta, v) & =\left(e, u_{0}\right) & & \text { in } I \times\{t=0\}, \\
\rho_{0}^{\gamma-1} & =0 & & \text { on } \Gamma,
\end{aligned}
$$

with $\rho_{0}^{\gamma-1}(x) \geq C \operatorname{dist}(x, \Gamma)$ for $x \in I$ near $\Gamma$.

\subsection{Setting $\gamma=2$}

We will begin our analysis of (1.10) by considering $\gamma=2$, in which case we seek solutions $\eta$ to the following system:

$$
\begin{aligned}
\rho_{0} v_{t}+\left(\rho_{0}^{2} / \eta_{x}^{2}\right)_{x} & =0 & & \text { in } I \times(0, T], \\
(\eta, v) & =\left(e, u_{0}\right) & & \text { on } I \times\{t=0\}, \\
\rho_{0} & =0 & & \text { on } \Gamma,
\end{aligned}
$$

with $\rho_{0}(x) \geq C \operatorname{dist}(x, \Gamma)$ for $x \in I$ near $\Gamma$. 
Equation (1.11a) is equivalent to

$$
v_{t}+2 \eta_{x}^{-1}\left(\rho_{0} \eta_{x}^{-1}\right)_{x}=0
$$

and (1.12) can be written as

$$
v_{t}+\rho_{0}\left(\eta_{x}^{-2}\right)_{x}+2\left(\rho_{0}\right)_{x} \eta_{x}^{-2}=0 .
$$

Because of the degeneracy caused by $\rho_{0}=0$ on $\Gamma$, all three equivalent forms of the compressible Euler equations are crucially used in our analysis. Equation (1.11a) is used for energy estimates, while (1.12) and (1.13) are used for additional elliptic-type estimates that rely on our higher-order Hardy-type inequality.

\subsection{Reference Domain $I$}

As we have already noted above, the initial domain $I \subset \mathbb{R}$ at time $t=0$ is given by

$$
I=(0,1),
$$

and the initial boundary points are given by $\Gamma=\partial I=\{0,1\}$.

\subsection{Higher-Order Energy Function for the Case $\gamma=2$}

We will consider the following higher-order energy function:

$$
\begin{aligned}
E(t, v)= & \sum_{s=0}^{4}\left\|\partial_{t}^{s} v(t, \cdot)\right\|_{H^{2-s / 2}(I)}^{2}+\sum_{s=0}^{2}\left\|\rho_{0} \partial_{t}^{2 s} v(t, \cdot)\right\|_{H^{3-s}(I)}^{2} \\
& +\left\|\sqrt{\rho_{0}} \partial_{t} \partial_{x}^{2} v(t, \cdot)\right\|_{L^{2}(I)}^{2}+\left\|\sqrt{\rho_{0}} \partial_{t}^{3} \partial_{x} v(t, \cdot)\right\|_{L^{2}(I)}^{2} .
\end{aligned}
$$

We define the polynomial function $M_{0}$ by

$$
M_{0}=P(E(0, v)),
$$

where $P$ denotes a generic polynomial function of its arguments.

\subsection{Main Result}

THEOREM 1.1 (Existence and Uniqueness for the Case $\gamma=2$ ) Given initial data $\left(u_{0}, \rho_{0}\right)$ such that $M_{0}<\infty, \rho_{0}(x)>0$ in $I$, and the physical vacuum condition (1.4) holds for $\rho_{0}$, there exists a solution to (1.11) (and hence (1.1)) on $[0, T]$ for $T>0$ taken sufficiently small such that

$$
\sup _{t \in[0, T]} E(t) \leq 2 M_{0}
$$

Moreover, if the initial data satisfies

$$
\sum_{s=0}^{3}\left\|\partial_{t}^{s} v(0, \cdot)\right\|_{3-s}^{2}+\sum_{s=0}^{3}\left\|\rho_{0} \partial_{t}^{2 s} v(0, \cdot)\right\|_{4-s}^{2}<\infty,
$$

then the solution is unique.

Remark 1.2. The case of arbitrary $\gamma>1$ is treated in Theorem 8.1 below. 
Remark 1.3. Given the regularity provided by the energy function (1.14), we see that the Lagrangian flow map $\eta \in C\left([0, T], H^{2}(I)\right)$. In our estimates for the multidimensional problem [1], we showed that $\eta$ gains regularity with respect to the velocity field $v$, and this fact is essential to control the geometry of the evolving free surface. This improved regularity for $\eta$ also holds in the one-dimensional setting, but it is not necessary for our estimates, as no geometry is involved.

Remark 1.4. Given $u_{0}$ and $\rho_{0}$, and using the fact that $\eta(x, 0)=x$, the quantity $\left.v_{t}\right|_{t=0}$ is computed using (1.11a):

$$
\left.v_{t}\right|_{t=0}=-\left.\left(\frac{1}{\rho_{0}}\left(\frac{\rho_{0}^{2}}{\eta_{x}^{2}}\right)_{x}\right)\right|_{t=0}=-2 \frac{\partial \rho_{0}}{\partial x} .
$$

Similarly, for all $k \in \mathbb{N}$,

$$
\left.\partial_{t}^{k} v\right|_{t=0}=-\left.\partial_{t}^{k-1}\left(\frac{1}{\rho_{0}}\left(\frac{\rho_{0}^{2}}{\eta_{x}^{2}}\right)_{x}\right)\right|_{t=0},
$$

so that each $\left.\partial_{t}^{k} v\right|_{t=0}$ is a function of space derivatives of $u_{0}$ and $\rho_{0}$.

Remark 1.5. Notice that our functional framework provides solutions that have optimal Sobolev regularity all the way to the boundary. Hence, in the physical case that $c \sim \sqrt{\operatorname{dist}(\partial I)}$, no singular behavior occurs near the vacuum boundary, even though both families of characteristics cross, and in particular, meet tangentially to $\Gamma(t)$ at a point.

Remark 1.6. Because of the degeneracy of the density function $\rho_{0}$ at the initial boundary $\partial I$, no compatibility conditions are required for the initial data.

\subsection{Prior Results for Compressible Euler Equations with Vacuum Boundary}

Some of the early developments in the theory of vacuum states for compressible gas dynamics can be found in $[8,11]$. We are aware of only a handful of previous theorems pertaining to the existence of solutions to the compressible and undamped Euler equations with moving vacuum boundary. Makino [14] considered compactly supported initial data and treated the compressible Euler equations for a gas as being set on $\mathbb{R}^{3} \times(0, T]$. With his methodology, it is not possible to track the location of the vacuum boundary (nor is it necessary); nevertheless, an existence theory was developed in this context, by a variable change that permitted the standard theory of symmetric hyperbolic systems to be employed. Unfortunately, the constraints on the data are too severe to allow for the evolution of the physical vacuum boundary.

In [9], Lindblad proved existence and uniqueness for the three-dimensional compressible Euler equations modeling a liquid rather than a gas. For a compressible liquid, the density $\rho \geq \lambda>0$ is assumed to be a strictly positive constant on the moving vacuum boundary $\Gamma(t)$ and is thus uniformly bounded from below by a positive constant. As such, the compressible liquid provides a uniformly hyperbolic, but characteristic, system. Lindblad used Lagrangian variables combined 
with Nash-Moser iteration to construct solutions. More recently Trakhinin [16] provided an alternative proof for the existence of a compressible liquid, employing a solution strategy based on symmetric hyperbolic systems combined with NashMoser iteration.

In the presence of damping, and with mild singularity, some existence results of smooth solutions are available, based on the adaptation of the theory of symmetric hyperbolic systems. In [12], a local existence theory was developed for the case that $c^{\alpha}$ (with $0<\alpha \leq 1$ ) is smooth across $\Gamma$, using methods that are not applicable to the local existence theory for the physical vacuum boundary. An existence theory for the small perturbation of a planar wave was developed in [17]. See also $[13,18]$ for other features of the vacuum state problem.

The only existence theory for the physical vacuum boundary condition that we know of can be found in the recent paper by Jang and Masmoudi [6] for the onedimensional compressible gas, wherein weighted Sobolev norms are used for the energy estimates. From these weighted norms, the regularity of the solutions cannot be directly determined. Letting $d$ denote the distance function to the boundary $\partial I$, and letting $\|\cdot\|_{0}$ denote the $L^{2}(I)$-norm, an example of the type of bound that is proved for the rescaled velocity field in [6] is the following:

$$
\begin{aligned}
&\|d v\|_{0}^{2}+\left\|d v_{x}\right\|_{0}^{2}+\left\|d v_{x x}+2 v_{x}\right\|_{0}^{2}+\left\|d v_{x x x}+2 v_{x x}-2 d^{-1} v_{x}\right\|_{0}^{2} \\
&+\left\|d v_{x x x x}+4 v_{x x x}-4 d^{-1} v_{x x}\right\|_{0}^{2}<\infty .
\end{aligned}
$$

The problem with inferring the regularity of $v$ from this bound can already be seen at the level of an $H^{1}(I)$-estimate. In particular, the bound on the norm $\| d v_{x x}+$ $2 v_{x} \|_{0}^{2}$ only implies a bound on $\left\|d v_{x x}\right\|_{0}^{2}$ and $\left\|v_{x}\right\|_{0}^{2}$ if the integration by parts on the cross-term,

$$
4 \int_{I} d v_{x x} v_{x}=-2 \int_{I} d_{x}\left|v_{x}\right|^{2}
$$

can be justified, which in turn requires having better regularity for $v_{x}$ than the a priori bounds provide. Any methodology that seeks regularity in (unweighted) Sobolev spaces for solutions must contend with this type of issue. We overcome this difficulty by constructing (sufficiently) smooth solutions to a degenerate parabolic regularization, and thus the sort of integration-by-parts difficulty just described is overcome. One can view our solutions as degenerate viscosity solutions. The key to their construction is our higher-order Hardy-type inequality that we provide below.

\section{Notation and Weighted Spaces}

\subsection{Differentiation and Norms in the Open Interval $I$}

Throughout the paper the symbol $D$ will be used to denote $\frac{\partial}{\partial x}$. 
For integers $k \geq 0$, we define the Sobolev space $H^{k}(I)$ to be the completion of $C^{\infty}(I)$ in the norm

$$
\|u\|_{k}:=\left(\sum_{a \leq k} \int_{\Omega}\left|D^{a} u(x)\right|^{2} d x\right)^{1 / 2}
$$

For real numbers $s \geq 0$, the Sobolev spaces $H^{s}(I)$ and the norms $\|\cdot\|_{s}$ are defined by interpolation. We use $H_{0}^{1}(I)$ to denote the subspace of $H^{1}(I)$ consisting of those functions $u(x)$ that vanish at $x=0$ and $x=1$.

\subsection{Embedding of a Weighted Sobolev Space}

Let $d$ denote the distance function to the boundary $\Gamma$, and let $p=1$ or 2 . Then the weighted Sobolev space $H_{d^{p}}^{1}(I)$, with norm given by $\int_{I} d(x)^{p}\left(|F(x)|^{2}+\right.$ $\left.|D F(x)|^{2}\right) d x$ for any $F \in H_{d^{p}}^{1}(I)$, satisfies the following embedding:

$$
H_{d^{p}}^{1}(I) \hookrightarrow H^{1-p / 2}(I),
$$

so that there is a constant $C>0$ depending only on $I$ and $p$ such that

$$
\|F\|_{1-p / 2}^{2} \leq C \int_{I} d(x)^{p}\left(|F(x)|^{2}+|D F(x)|^{2}\right) d x .
$$

See, for example, [7, sec. 8].

\subsection{Sobolev Embedding and Interpolation Inequalities}

For $s<\frac{1}{2}$,

$$
\|u\|_{L^{2 /(1-2 s)}(I)} \leq C_{s}\|u\|_{s} .
$$

We will also make use of the standard interpolation inequality:

$$
\|u\|_{3 / 4} \leq C\|u\|_{1 / 2}^{1 / 2}\|u\|_{1}^{1 / 2} \text {. }
$$

\section{A Higher-Order Hardy-Type Inequality}

We will make fundamental use of the following generalization of the wellknown Hardy inequality to higher-order derivatives:

LEMMA 3.1 (Higher-Order Hardy-Type Inequality) Let $s \geq 1$ be a given integer, and suppose that

$$
u \in H^{S}(I) \cap H_{0}^{1}(I) .
$$

If $d(x)>0$ for $x \in I, d \in H^{r}(I), r=\max (s-1,2)$, and $d$ is the distance function to $\partial I$ near $\partial I$, then $\frac{u}{d} \in H^{s-1}(I)$ with

$$
\left\|\frac{u}{d}\right\|_{s-1} \leq C\|u\|_{s} .
$$


ProOF: We use an induction argument. The case $s=1$ is of course the classical Hardy inequality. Let us now assume that inequality (3.1) holds for a given $s \geq 1$, and suppose that

$$
u \in H^{s+1}(I) \cap H_{0}^{1}(I) .
$$

Using $D$ to denote $\frac{\partial}{\partial x}$, a simple computation shows that for $m \in \mathbb{N}$,

$$
D^{m}\left(\frac{u}{d}\right)=\frac{f}{d^{m+1}}
$$

with

$$
f=\sum_{k=0}^{m} C_{m}^{k} D^{m-k} u k !(-1)^{k} d^{m-k}
$$

for a constant $C_{m}^{k}$ depending on $k$ and $m$. From the regularity of $u$, we see that $f \in H_{0}^{1}(I)$. Next, with $D=\frac{\partial}{\partial x}$, we obtain the identity

$$
\begin{aligned}
D f= & \sum_{k=0}^{s} C_{s}^{k} D^{s+1-k} u k !(-1)^{k} d^{s-k} \\
& +\sum_{k=0}^{s-1} C_{s}^{k} D^{s-k} u k !(-1)^{k} d^{s-k-1}(s-k) \\
= & D^{s+1} u s !(-1)^{s} d^{s}+\sum_{k=1}^{s} C_{s}^{k} D^{s+1-k} u k !(-1)^{k} d^{s-k} \\
& +\sum_{k=0}^{s-1} C_{s}^{k+1} D^{s-k} u(k+1) !(-1)^{k} d^{s-k-1} \\
= & D^{s+1} u s !(-1)^{s} d^{s} .
\end{aligned}
$$

Since $f \in H_{0}^{1}(I)$, we deduce from (3.3) that for any $x \in\left(0, \frac{1}{2}\right]$,

$$
f(x)=(-1)^{s} s ! \int_{0}^{x} D^{s+1} u(y) y^{s} d y
$$

which by substitution in (3.2) yields the identity

$$
D^{s}\left(\frac{u}{d}\right)(x)=\frac{(-1)^{s} s ! \int_{0}^{x} D^{s+1} u(y) y^{s} d y}{x^{s+1}},
$$

which by a simple majoration provides the bound

$$
\left|D^{s}\left(\frac{u}{d}\right)(x)\right| \leq s ! \frac{\psi_{1}(x) \int_{0}^{x}\left|D^{s+1} u(y)\right| d y}{d(x)},
$$

where $\psi_{1}$ is the piecewise affine function equal to 1 on $\left[0, \frac{1}{2}\right]$ and to 0 on $\left[\frac{3}{4}, 1\right]$. 
Next, for any $x \in\left[\frac{1}{2}, 1\right)$, we similarly obtain that

$$
\left|D^{s}\left(\frac{u}{d}\right)(x)\right| \leq s ! \frac{\psi_{2}(x) \int_{x}^{1}\left|D^{s+1} u(y)\right| d y}{d(x)},
$$

where $\psi_{2}$ is the piecewise affine function equal to 0 on $\left[0, \frac{1}{4}\right]$ and to 1 on $\left[\frac{1}{2}, 1\right]$, so that for any $x \in I$ :

$$
\left|D^{s}\left(\frac{u}{d}\right)(x)\right| \leq s ! \frac{\psi_{1}(x) \int_{0}^{x}\left|D^{s+1} u(y)\right| d y+\psi_{2}(x) \int_{x}^{1}\left|D^{s+1} u(y)\right| d y}{d(x)}
$$

Now, with $g=\psi_{1}(x) \int_{0}^{x}\left|D^{s+1} u(y)\right| d y+\psi_{2}(x) \int_{x}^{1}\left|D^{s+1} u(y)\right| d y$, we notice that $g \in H_{0}^{1}(I)$, with

$$
\|g\|_{1} \leq C\left\|D^{s+1} u\right\|_{0} .
$$

Therefore, by the classical Hardy inequality, we infer from (3.4) that

$$
\left\|D^{s}\left(\frac{u}{d}\right)\right\|_{0} \leq C\|g\|_{1} \leq C\left\|D^{s+1} u\right\|_{0} .
$$

Since we assumed in our induction process that our generalized Hardy inequality is true at order $s$, we then have that

$$
\left\|\frac{u}{d}\right\|_{s-1} \leq C\|u\|_{s}
$$

which, together with (3.5), implies that

$$
\left\|\frac{u}{d}\right\|_{s} \leq C\|u\|_{s+1},
$$

and thus establishes the property at order $s+1$ and concludes the proof.

In order to obtain estimates independent of a regularization parameter $\kappa$ defined in Section 4, we will also need the following lemma, whose proof can be found in [2, lemma 1, sec. 6]:

LEMMA 3.2 Let $\kappa>0$ and $\left.g \in L^{\infty}\left(0, T ; H^{s}(I)\right)\right)$ be given, and let $f \in$ $H^{1}\left(0, T ; H^{s}(I)\right)$ be such that

$$
f+\kappa f_{t}=g \quad \text { in }(0, T) \times I .
$$

Then,

$$
\|f\|_{L^{\infty}\left(0, T ; H^{s}(I)\right)} \leq C \max \left\{\|f(0)\|_{s},\|g\|_{L^{\infty}\left(0, T ; H^{s}(I)\right)}\right\} .
$$




\section{An Asymptotically Consistent Degenerate Parabolic $\kappa$-Approximation of the Compressible Euler Equations in Vacuum}

\subsection{Smoothing the Initial Data}

For the purpose of constructing solutions, we will smooth the initial velocity field $u_{0}$. We will also smooth the initial density field $\rho_{0}$ while preserving the conditions that $\rho(x)>0$ for $x \in I$ and that $\rho_{0}$ satisfies (1.4) near $\Gamma$.

For $\kappa>0$, let $0 \leq \varrho_{\kappa} \in C_{0}^{\infty}(\mathbb{R})$ denote the standard family of mollifiers with $\operatorname{spt}\left(\varrho_{\kappa}\right) \subset \overline{B(0, \kappa)}$, and let $\mathcal{E}_{I}$ denote a Sobolev extension operator mapping $H^{s}(I)$ to $H^{s}(\mathbb{R})$ for $s \geq 0$.

For $\kappa \in\left(0, \frac{1}{2}\right)$, we set

$$
u_{0}^{\kappa}=\varrho_{1|\ln \kappa|} * \mathcal{E}_{I}\left(u_{0}\right),
$$

so that for any fixed $\kappa \in\left(0, \frac{1}{2}\right), u_{0}^{\kappa} \in C^{\infty}(\bar{\Omega})$. More importantly, due to the standard properties of convolution, we also have the following estimates:

$$
\forall s \geq 1, \forall \kappa \in\left(0, \frac{1}{2}\right), \quad\left\|u_{0}^{\kappa}\right\|_{s} \leq C_{s} *|\kappa|^{s}\left\|u_{0}\right\|_{0} .
$$

The smoothed initial density function $\rho_{0}^{\kappa}$ is defined as the solution of the elliptic equation

$$
\begin{aligned}
\partial_{x}^{2} \rho_{0}^{\kappa} & =\partial_{x}^{2}\left[\varrho_{1 /|\ln \kappa|} * \mathcal{E}_{I}\left(\rho_{0}\right)\right] & & \text { in } I, \\
\rho_{0}^{\kappa} & =0 & & \text { on } \Gamma .
\end{aligned}
$$

By elliptic regularity, $\rho_{0}^{\kappa} \in C^{\infty}(\bar{\Omega})$, and by choosing $\kappa>0$ sufficiently small, we see that $\rho_{0}^{\kappa}(x)>0$ for $x \in \Omega$, and that the physical vacuum condition (1.4) is satisfied near $\Gamma$. This follows from the fact that $\partial_{x} \rho_{0}^{\kappa}<0$ on $\Gamma$ for $\kappa>0$ taken sufficiently small, which implies that $\rho_{0}^{\kappa}(x)>0$ for $x \in I$ very close to $\Gamma$. On the other hand, $\rho_{0}^{\kappa}(x)>0$ for all $x \in J$ for any open subset $J \subset I$ by taking $\kappa$ sufficiently small. We also have the estimate

$$
\forall s \geq 1, \forall \kappa \in\left(0, \frac{1}{2}\right), \quad\left\|\rho_{0}^{\kappa}\right\|_{s} \leq C_{s}|\ln \kappa|^{s}\left\|\rho_{0}\right\|_{0} .
$$

Until Section 7.4, for notational convenience, we will denote $u_{0}^{\kappa}$ by $u_{0}$ and $\rho_{0}^{\kappa}$ by $\rho_{0}$. In Section 7.4, we will show that Theorem 1.1 holds with the optimal regularity stated therein.

\subsection{Degenerate Parabolic Approximation to Compressible Euler Equations: The $\kappa$-Problem}

For $\kappa>0$, we consider the following nonlinear degenerate parabolic approximation of the compressible Euler system (1.11):

$$
\begin{aligned}
\rho_{0} v_{t}+\left(\rho_{0}^{2} / \eta_{x}^{2}\right)_{x} & =\kappa\left[\rho_{0}^{2} v_{x}\right]_{x} & & \text { in } I \times(0, T], \\
(\eta, v) & =\left(e, u_{0}\right) & & \text { on } I \times\{t=0\}, \\
\rho_{0} & =0 & & \text { on } \Gamma,
\end{aligned}
$$


with the smoothed $u_{0}$ given by (4.1), and the smoothed $\rho_{0}$ given by (4.3) and thus satisfying the physical vacuum condition (1.4).

We will first obtain the existence of a solution to (4.5) on a short time interval $\left[0, T_{\kappa}\right]$ (with $T_{\kappa}$ depending a priori on $\kappa$ ). We will then perform energy estimates on this solution that will show that the time of existence, in fact, does not depend on $\kappa$, and moreover that our a priori estimates for this sequence of solutions are also independent of $\kappa$. The existence of a solution to the compressible Euler equations (1.11) is then obtained as the weak limit as $\kappa \rightarrow 0$ of the sequence of solutions to $(4.5)$.

\section{Solving the Parabolic $\kappa$-Problem (4.5) by a Fixed-Point Method}

For notational convenience, we will write

$$
\eta^{\prime}=\frac{\partial \eta}{\partial x}
$$

and similarly for other functions.

\subsection{Assumption on Initial Data}

Given $u_{0}$ and $\rho_{0}$ and using the fact that $\eta(x, 0)=x$, we can compute the quantity $\left.v_{t}\right|_{t=0}$ for the degenerate parabolic $\kappa$-problem by using (4.5a):

$$
\left.v_{t}\right|_{t=0}=\left.\left(\frac{\kappa}{\rho_{0}}\left[\rho_{0}^{2} v^{\prime}\right]^{\prime}-\frac{1}{\rho_{0}}\left(\frac{\rho_{0}^{2}}{\eta^{\prime 2}}\right)^{\prime}\right)\right|_{t=0}=\left(\frac{\kappa}{\rho_{0}}\left[\rho_{0}^{2} u_{0}^{\prime}\right]^{\prime}-2 \rho_{0}^{\prime}\right) .
$$

Similarly, for all $k \in \mathbb{N}$,

$$
u_{k}:=\left.\partial_{t}^{k} v\right|_{t=0}=\left.\partial_{t}^{k-1}\left(\frac{\kappa}{\rho_{0}}\left[\rho_{0}^{2} v^{\prime}\right]^{\prime}-\frac{1}{\rho_{0}}\left(\frac{\rho_{0}^{2}}{\eta^{\prime 2}}\right)^{\prime}\right)\right|_{t=0} .
$$

These formulae make it clear that each $\left.\partial_{t}^{k} v\right|_{t=0}$ is a function of space derivatives of $u_{0}$ and $\rho_{0}$. In the following, we will mostly use the notation $\left.\partial_{t}^{k} v\right|_{t=0}$ for time derivatives evaluated at $t=0$, as it is more descriptive than $u_{k}$.

\subsection{Functional Framework for the Fixed-Point Scheme and Some Notational Conventions}

For $T>0$, we shall denote by $\mathcal{X}_{T}$ the following Hilbert space:

$$
\begin{aligned}
\mathcal{X}_{T}=\{v & \in L^{2}\left(0, T ; H^{2}(I)\right) \mid \\
& \partial_{t}^{4} v \in L^{2}\left(0, T ; H^{1}(I)\right), \rho_{0} \partial_{t}^{4} v \in L^{2}\left(0, T ; H^{2}(I)\right), \\
& \left.\partial_{t}^{3} v \in L^{2}\left(0, T ; H^{2}(I)\right), \rho_{0} \partial_{t}^{3} v \in L^{2}\left(0, T ; H^{3}(I)\right)\right\},
\end{aligned}
$$

endowed with its natural Hilbert norm

$$
\begin{aligned}
\|v\|_{\mathcal{X}_{T}}^{2}= & \|v\|_{L^{2}\left(0, T ; H^{2}(I)\right)}^{2}+\left\|\partial_{t}^{4} v\right\|_{L^{2}\left(0, T ; H^{1}(I)\right)}^{2}+\left\|\rho_{0} \partial_{t}^{4} v\right\|_{L^{2}\left(0, T ; H^{2}(I)\right)}^{2} \\
& +\left\|\partial_{t}^{3} v\right\|_{L^{2}\left(0, T ; H^{2}(I)\right)}^{2}+\left\|\rho_{0} \partial_{t}^{3} v\right\|_{L^{2}\left(0, T ; H^{3}(I)\right)}^{2} .
\end{aligned}
$$


For $M>0$ to be made precise below, we define the following closed, bounded, convex subset of $\mathcal{X}_{T}$ :

$$
\mathcal{C}_{T}(M)=\left\{v \in \mathcal{X}_{T}:\left.\partial_{t}^{a} v\right|_{t=0}=u_{a} \text { for } a=0,1,2,3,\|v\|_{\mathcal{X}_{T}} \leq M\right\},
$$

where $u_{a}$ is defined in (5.1), and where $\mathcal{C}_{T}(M)$ is indeed nonempty if $M$ is large enough. Henceforth, we assume that $T>0$ is given such that independently of the choice of $v \in \mathcal{C}_{T}(M)$,

$$
\eta(x, t)=x+\int_{0}^{t} v(x, s) d s
$$

is injective for $t \in[0, T]$, and that $\frac{1}{2} \leq \eta^{\prime}(x, t) \leq \frac{3}{2}$ for $t \in[0, T]$ and $x \in \bar{I}$. This can be achieved by taking $T>0$ sufficiently small: with $e(x)=x$ so that $e^{\prime}(x)=1$, notice that

$$
\left\|\eta^{\prime}(\cdot, t)-1\right\|_{1}=\left\|\int_{0}^{t} v^{\prime}(\cdot, s) d s\right\|_{1} \leq \sqrt{T} M .
$$

The space $\mathcal{X}_{T}$ will be appropriate for our fixed-point methodology to prove existence of a solution to our $\kappa$-regularized parabolic problem (4.5).

Finally, we define a polynomial function $\mathcal{N}_{0}$ of norms of the nonsmoothed initial data $u_{0}$ and $\rho_{0}$ as follows:

$$
\mathcal{N}_{0}=P_{\kappa}\left(\left\|u_{0}\right\|_{0},\left\|\rho_{0}\right\|_{0}\right),
$$

where $P_{\kappa}$ is a generic polynomial with coefficients depending on powers of $|\ln \kappa|$. We then set

$$
M=\mathcal{N}_{0}+1 .
$$

Thanks to the properties of the convolution (4.2) and (4.4), we then have for the quantities defined in (5.1) (using the smoothed initial data $u_{0}^{\kappa}$ and $\rho_{0}^{\kappa}$ ):

$$
\forall s \geq 1, \forall k \in\{0,1,2,3,4,5\},\left\|\partial_{t}^{k} v(0)\right\|_{s} \leq C_{s} P_{\kappa}\left(\left\|u_{0}\right\|_{0},\left\|\rho_{0}\right\|_{0}\right) \leq \mathcal{N}_{0} .
$$

As the coefficients of $P_{\kappa}$ depend on powers of $|\ln \kappa|$, we then see that

$$
\begin{gathered}
\forall s \geq 1, \forall k \in\{0,1,2,3,4,5\}, \\
\kappa\left\|\partial_{t}^{k} v(0)\right\|_{s} \leq C_{s} \sqrt{\kappa} P\left(\left\|u_{0}\right\|_{0},\left\|\rho_{0}\right\|_{0}\right) \rightarrow 0 \quad \text { as } \kappa \rightarrow 0 .
\end{gathered}
$$

where $P$ is a polynomial with coefficients independent of $\kappa$, and where we have the fact that $\kappa|\ln \kappa|^{S} \leq \sqrt{\kappa}$ for any power $s \geq 0$ and $\kappa$ small enough.

\subsection{Theorem for Existence and Uniqueness of Solutions to the Parabolic $\kappa$-Problem}

We will make use of the Tychonoff fixed-point theorem in our fixed-point procedure (see, for example, [5]). Recall that this states that for a reflexive separable Banach space $\mathcal{X}_{T}$ and $\mathcal{C}_{T}(M) \subset \mathcal{X}_{T}$ a closed, convex, bounded subset, if $F: \mathcal{C}_{T}(M) \rightarrow \mathcal{C}_{T}(M)$ is weakly sequentially continuous into $\mathcal{X}_{T}$, then $F$ has at least one fixed point. 
THEOREM 5.1 (Solutions to Parabolic $\kappa$-Problem) Given our smooth data, for $T>0$ taken sufficiently small, there exists a unique solution $v \in \mathcal{X}_{T}$ to the degenerate parabolic $\kappa$-problem (4.5).

\subsection{Linearizing the Degenerate Parabolic $\kappa$-Problem}

Given $\bar{v} \in \mathcal{C}_{T}(M)$ and defining $\bar{\eta}(x, t)=x+\int_{0}^{t} \bar{v}(x, \tau) d \tau$, we consider the linear equation for $v$ :

$$
\rho_{0} v_{t}+\left[\frac{\rho_{0}^{2}}{\bar{\eta}^{\prime 2}}\right]^{\prime}-\kappa\left[\rho_{0}^{2} v^{\prime}\right]^{\prime}=0 .
$$

We will prove the following:

(1) $v$ is a unique solution to (5.7);

(2) $v \in \mathcal{C}_{T}(M)$ for $T$ taken sufficiently small;

(3) the map $\bar{v} \mapsto v: \mathcal{C}_{T}(M) \rightarrow \mathcal{C}_{T}(M)$ and is sequentially weakly continuous in $\mathcal{X}_{T}$.

The solution to our parabolic $\kappa$-problem (4.5) will then be obtained as a fixed point of the map $\bar{v} \mapsto v$ in $\mathcal{X}_{T}$ via the Tychonoff fixed-point theorem.

In order to use our higher-order Hardy-type inequality, Lemma 3.1, it will be convenient to introduce the new variable

$$
X=\rho_{0} v^{\prime}
$$

which then belongs to $H_{0}^{1}(I)$. By a simple computation, we see that (5.7) is equivalent to

$$
v_{t}^{\prime}+\left[\frac{2}{\bar{\eta}^{\prime}}\left(\frac{\rho_{0}}{\bar{\eta}^{\prime}}\right)^{\prime}\right]^{\prime}-\kappa\left[\frac{1}{\rho_{0}}\left[\rho_{0}^{2} v^{\prime}\right]^{\prime}\right]^{\prime}=0,
$$

and hence that

$$
\begin{aligned}
\frac{X_{t}}{\rho_{0}}-\kappa\left[\frac{1}{\rho_{0}}\left(\rho_{0} X\right)^{\prime}\right]^{\prime} & =-\left[\frac{2}{\bar{\eta}^{\prime}}\left(\frac{\rho_{0}}{\bar{\eta}^{\prime}}\right)^{\prime}\right]^{\prime} & & \text { in }[0, T] \times I, \\
X & =0 & & \text { on }[0, T] \times \partial I, \\
\left.X\right|_{t=0} & =\rho_{0} u_{0}^{\prime} & & \text { on } I .
\end{aligned}
$$

We shall therefore solve the degenerate linear parabolic problem (5.8) with Dirichlet boundary conditions, which (as we will prove) will surprisingly admit a solution with arbitrarily high space regularity (depending on the regularity of the right-hand side and the initial data, of course), and not just an $H_{0}^{1}(I)$-type weak solution. After we obtain the solution $X$, we will then easily find our solution $v$ to (5.7).

In order to construct our fixed point, we will need to obtain estimates for $X$ (and hence $v$ ) with high space regularity; in particular, we will need to study the fifth time-differentiated problem. For this purpose, it is convenient to define the new variable

$$
Y=\partial_{t}^{5} X=\rho_{0} \partial_{t}^{5} v^{\prime}
$$


and consider the following equation for $Y$ :

$$
\begin{aligned}
\frac{Y_{t}}{\rho_{0}}-\kappa\left[\frac{1}{\rho_{0}}\left(\rho_{0} Y\right)^{\prime}\right]^{\prime} & =-\partial_{t}^{5}\left[\frac{2}{\bar{\eta}^{\prime}}\left(\frac{\rho_{0}}{\bar{\eta}^{\prime}}\right)^{\prime}\right]^{\prime} & & \text { in }[0, T] \times I, \\
Y & =0 & & \text { on }[0, T] \times \partial I, \\
\left.Y\right|_{t=0} & =Y_{\text {init }} & & \text { in } I,
\end{aligned}
$$

where $Y_{\text {init }}=\left.\rho_{0} \partial_{t}^{5} v^{\prime}\right|_{t=0}$.

\subsection{Existence of a Weak Solution to Linear Problem (5.10) by a Galerkin Scheme}

Let $\left(e_{n}\right)_{n \in \mathbb{N}}$ denote a Hilbert basis of $H_{0}^{1}(I)$, with each $e_{n}$ being of class $H^{2}(I)$. Such a choice of basis is indeed possible as we can take for instance the eigenfunctions of the Laplace operator on $I$ with vanishing Dirichlet boundary conditions. We then define the Galerkin approximation at order $n \geq 1$ of (5.8) as being under the form $Y_{n}=\sum_{i=0}^{n} \lambda_{i}^{n}(t) e_{i}$ such that $\forall k \in\{0, \ldots, n\}$,

$$
\begin{gathered}
\left(\frac{Y_{n t}}{\rho_{0}}, e_{k}\right)_{L^{2}(I)}+\kappa\left(\left(\rho_{0} Y_{n}\right)^{\prime}, \frac{e_{k}^{\prime}}{\rho_{0}}\right)_{L^{2}(I)}= \\
\left(\partial_{t}^{5}\left[\frac{2}{\bar{\eta}^{\prime}}\left[\frac{\rho_{0}}{\bar{\eta}^{\prime}}\right]^{\prime}\right] e_{k}^{\prime}\right)_{L^{2}(I)} \text { in }[0, T], \\
\lambda_{i}^{n}(0)=\left(Y_{\text {init }}, e_{i}\right)_{L^{2}(I)} .
\end{gathered}
$$

Since each $e_{i}$ is in $H^{k}(I) \cap H_{0}^{1}(I)$ for all $k \geq 1$, we have by our high-order Hardy-type inequality (3.1) that

$$
\frac{e_{i}}{\rho_{0}} \in H^{k-1}(I) \text { for all } k \geq 1 \text {; }
$$

therefore, each integral written in (5.11a) is well-defined. Furthermore, as the $e_{i}$ are linearly independent, so are the $e_{i} / \sqrt{\rho_{0}}$ and therefore the determinant of the matrix

$$
\left(\left(\frac{e_{i}}{\sqrt{\rho_{0}}}, \frac{e_{j}}{\sqrt{\rho_{0}}}\right)_{L^{2}(I)}\right)_{(i, j) \in \mathbb{N}_{n}=\{0,1, \ldots, n\}}
$$

is nonzero. This implies that (5.11a), our finite-dimensional Galerkin approximation, is a well-defined first-order differential system of order $n+1$, which therefore has a solution on a time interval $\left[0, T_{n}\right]$, where $T_{n}$ a priori depends on the dimension $n$ of the Galerkin approximation. In order to prove that $T_{n}=T$, with $T$ independent of $n$, we notice that since $Y_{n}$ is a linear combination of the $e_{i}\left(i \in \mathbb{N}_{n}\right)$, we have that

$$
\left(\frac{Y_{n t}}{\rho_{0}}-\kappa\left[\frac{1}{\rho_{0}}\left(\rho_{0} Y_{n}\right)^{\prime}\right]^{\prime}, Y_{n}\right)_{L^{2}(I)}=\left(\partial_{t}^{5}\left[\frac{2}{\bar{\eta}^{\prime}}\left[\frac{\rho_{0}}{\bar{\eta}^{\prime}}\right]^{\prime}\right] Y_{n}^{\prime}\right)_{L^{2}(I)}
$$


Since $Y_{n} \in H_{0}^{1}(I)$ and $\frac{1}{\rho_{0}}\left(\rho_{0} Y_{n}\right)^{\prime} \in H^{1}(I)$, integration by parts yields

$$
-\int_{I}\left[\frac{1}{\rho_{0}}\left(\rho_{0} Y_{n}\right)^{\prime}\right]^{\prime} Y_{n}=\int_{I}\left[\frac{1}{\rho_{0}}\left(\rho_{0} Y_{n}\right)^{\prime}\right] Y_{n}^{\prime}=\int_{I} Y_{n}^{\prime 2}+\int_{I} \rho_{0}^{\prime} \frac{Y_{n}}{\rho_{0}} Y_{n}^{\prime} .
$$

Next, using our higher-order Hardy-type inequality, we see that $\frac{Y_{n}}{\rho_{0}} \in H^{1}(I)$, and thus

$$
\int_{I} \rho_{0}^{\prime} \frac{Y_{n}}{\rho_{0}} Y_{n}^{\prime}=-\int_{I} \rho_{0}^{\prime} \frac{1}{\rho_{0}} Y_{n}^{\prime} Y_{n}+\int_{I} \frac{\rho_{0}^{\prime 2}}{\rho_{0}^{2}} Y_{n}^{2}-\int_{I} \rho_{0}^{\prime \prime} \frac{Y_{n}^{2}}{\rho_{0}},
$$

which implies that

$$
\int_{I} \rho_{0}^{\prime} \frac{Y_{n}}{\rho_{0}} Y_{n}^{\prime}=\frac{1}{2} \int_{I} \frac{\rho_{0}^{\prime 2}}{\rho_{0}^{2}} Y_{n}^{2}-\frac{1}{2} \int_{I} \rho_{0}^{\prime \prime} \frac{Y_{n}^{2}}{\rho_{0}} .
$$

Substitution of this identity into (5.12) and (5.13) yields

$$
\begin{aligned}
\frac{1}{2}\left[\frac{d}{d t} \int_{I} \frac{Y_{n}^{2}}{\rho_{0}}-\kappa \int_{I} \rho_{0}^{\prime \prime} \frac{Y_{n}^{2}}{\rho_{0}}\right]+\kappa \int_{I} Y_{n}^{\prime 2}+\frac{1}{2} \kappa \int_{I} \frac{\rho_{0}^{\prime 2}}{\rho_{0}^{2}} Y_{n}^{2}= \\
\quad-\int_{I} \partial_{t}^{5}\left[\frac{2}{\bar{\eta}^{\prime}}\left[\frac{\rho_{0}}{\bar{\eta}^{\prime}}\right]^{\prime}\right] Y_{n}^{\prime},
\end{aligned}
$$

which shows that (since our given $\bar{v} \in \mathcal{C}_{T}(M)$ )

$$
\begin{aligned}
\frac{d}{d t} \int_{I} \frac{Y_{n}^{2}}{\rho_{0}}-\kappa\left\|\rho_{0}^{\prime \prime}\right\|_{L^{\infty}} \int_{I} \frac{Y_{n}^{2}}{\rho_{0}}+\kappa \int_{I} Y_{n}^{\prime 2}+\kappa \int_{I} \rho_{0}^{\prime 2} \frac{Y_{n}^{2}}{\rho_{0}^{2}} \leq \\
C_{\kappa}\left\|\partial_{t}^{5}\left[\frac{2}{\bar{\eta}^{\prime}}\left[\frac{\rho_{0}}{\bar{\eta}^{\prime}}\right]^{\prime}\right]\right\|_{0}^{2}
\end{aligned}
$$

for a constant $C_{\kappa}$ depending on $\frac{1}{\kappa}$. Consequently, $T_{n}=T$ with $T$ independent of $n$, and

$$
\sup _{[0, T]} \int_{I} \frac{Y_{n}^{2}}{\rho_{0}}+\kappa \int_{0}^{T} \int_{I} Y_{n}^{\prime 2} \leq C_{\kappa} C_{M}+C \mathcal{N}_{0}
$$

where $C_{M}$ is a constant that depends on $M$, and where $\mathcal{N}_{0}$ is defined in (5.4). Thus, there exists a subsequence of $\left(Y_{n}\right)$ that converges weakly to some $Y \in$ $L^{2}\left(0, T ; H_{0}^{1}(I)\right)$, which satisfies

$$
\sup _{[0, T]} \int_{I} \frac{Y^{2}}{\rho_{0}}+\kappa \int_{0}^{T} \int_{I} Y^{\prime 2} \leq C_{\kappa} C_{M}+C \mathcal{N}_{0}
$$

With (5.9), we see that

$$
\left\|\rho_{0} \partial_{t}^{5} v^{\prime}\right\|_{L^{2}\left(0, T ; H^{1}(I)\right)} \leq C_{\kappa} C_{M}+C \mathcal{N}_{0} .
$$


Note that $\rho_{0} \partial_{t}^{5} v^{\prime}$ is not in our fixed-point space $\mathcal{X}_{T}$ and does not need a small bound; on the other hand, this estimate will provide a small bound (for short time) for the quantity $\rho_{0} \partial_{t}^{4} v^{\prime}$, which is part of our fixed-point space $\mathcal{X}_{T}$.

Furthermore, it can also be shown using standard arguments that $Y$ is a solution of (5.10) (where (5.10a) is satisfied almost everywhere in $[0, T] \times I$ and holds in a variational sense for all test functions in $\left.L^{2}\left(0, T ; H_{0}^{1}(I)\right)\right)$, and that

$$
\frac{Y_{t}}{\rho_{0}} \in L^{2}\left(0, T ; H^{-1}(I)\right) \text {. }
$$

Now, with the functions

$$
X_{i}=\left.\rho_{0} \frac{\partial^{i} v^{\prime}}{\partial t^{i}}\right|_{t=0} \text { for } i=0,1,2,3,4,
$$

we define

$$
\begin{aligned}
Z(t, x)=\int_{0}^{t} Y(\cdot, x) & =\rho_{0}(x) \int_{0}^{t} \partial_{t}^{5} v^{\prime}(\cdot, x) \\
& =\rho_{0}(x) \partial_{t}^{4} v^{\prime}(t, x)-X_{4}(x)
\end{aligned}
$$

and

$$
X(t, x)=\sum_{i=0}^{4} \frac{X_{i} t^{i}}{i !}+\int_{0}^{t} \int_{0}^{t_{4}} \int_{0}^{t_{3}} \int_{0}^{t_{2}} Z\left(t_{1}, x\right) d t_{1} d t_{2} d t_{3} d t_{4} .
$$

We then see that $X \in C^{0}\left([0, T] ; H_{0}^{1}(I)\right)$ is a solution of (5.8), with $\partial_{t}^{5} X=Y$. In order to obtain a fixed point for the map $\bar{v} \mapsto v$, we need to establish better space regularity for $Z$, and hence $X$ and $v$.

\subsection{Improved Space Regularity for $Z$}

We introduce the variable $\breve{v}$ defined by

$$
\check{v}(t, x)=\int_{0}^{x} \frac{X(t, \cdot)}{\rho_{0}(\cdot)},
$$

so that $\check{v}$ vanishes at $x=0$, and hence will allow us to employ the Poincaré inequality with this variable; note that it is not necessary to have $\breve{v}$ vanish at $x=1$ as well, as the condition $\breve{v}=0$ at $x=0$ suffices for the Poincaré inequality to hold. For this reason, it is more convenient to work with the variable $\breve{v}$ rather than $v$ itself.

It is easy to see that

$$
X=\rho_{0} \check{v}^{\prime}
$$

and

$$
Z=\rho_{0} \partial_{t}^{4} \check{v}^{\prime}
$$

Thanks to the standard Hardy inequality, we thus have that

$$
\left\|\partial_{t}^{4} \check{v}^{\prime}\right\|_{0} \leq C\|Z\|_{1},
$$


and hence by Poincaré's inequality,

$$
\left\|\partial_{t}^{4} \check{v}(t, \cdot)\right\|_{1} \leq C\|Z(t, \cdot)\|_{1}
$$

With

$$
F_{0}=\frac{Y_{\text {init }}}{\rho_{0}}+\left.\partial_{t}^{4}\left[\frac{2}{\bar{\eta}^{\prime}}\left[\frac{\rho_{0}}{\bar{\eta}^{\prime}}\right]^{\prime}\right]^{\prime}\right|_{t=0},
$$

our starting point is the equation

$$
\frac{Y}{\rho_{0}}-\kappa\left[\frac{1}{\rho_{0}}\left(\rho_{0} Z\right)^{\prime}\right]^{\prime}=-\partial_{t}^{4}\left[\frac{2}{\bar{\eta}^{\prime}}\left[\frac{\rho_{0}}{\bar{\eta}^{\prime}}\right]^{\prime}\right]^{\prime}+F_{0} \quad \text { in }[0, T] \times I,
$$

which follows from our definition of $Z$ given in (5.17) and time integration of (5.10a). From this equation, we infer that

$$
\kappa\left\|\left[\frac{1}{\rho_{0}}\left(\rho_{0} Z\right)^{\prime}\right]^{\prime}\right\|_{0} \leq\left\|\partial_{t}^{4}\left[\frac{2}{\bar{\eta}^{\prime}}\left[\frac{\rho_{0}}{\bar{\eta}^{\prime}}\right]^{\prime}\right]^{\prime}\right\|_{0}+\left\|\frac{Y}{\rho_{0}}\right\|_{0}+\left\|F_{0}\right\|_{0} .
$$

By the standard Hardy inequality and the fact that $\bar{v} \in \mathcal{C}_{T}(M)$, we obtain the estimate

$$
\kappa\left\|\left[\frac{1}{\rho_{0}}\left(\rho_{0} Z\right)^{\prime}\right]^{\prime}\right\|_{0} \leq C_{M}+\left\|\rho_{0} \bar{v}_{t t t}^{\prime \prime \prime}(t)\right\|_{0}+\left\|\bar{v}_{t t t}^{\prime \prime}(t)\right\|_{0} C\|Y\|_{1}+\mathcal{N}_{0},
$$

where $C_{M}$ is a constant that depends on $M$, and where the second and third terms on the right, $\left\|\rho_{0} \bar{v}_{t t t}^{\prime \prime \prime}(t)\right\|_{0}$ and $\left\|\bar{v}_{t t t}^{\prime \prime}(t)\right\|_{0}$, will be absorbed by $C_{M}$ once we integrate in time. The remaining terms are lower-order and are bounded by $C_{M}$; for example, one such term is given by $\left\|\ell(t) \rho_{0} \bar{v}_{t t}^{\prime \prime}(t) \bar{v}^{\prime}(t)\right\|_{0}$, where $\ell(t)$ is in $L^{\infty}(I)$. Given that $\left\|\rho_{0} \bar{v}_{t t t}\right\|_{L^{2}\left(0, T ; H^{3}(I)\right.}$ is bounded by $M$, by the fundamental theorem of calculus, we see that $\sup _{t \in[0, T]}\left\|\rho_{0} \bar{v}_{t t}^{\prime \prime}(t)\right\|_{0} \leq \mathcal{N}_{0}+C T M$, so that Hölder's inequality shows that $\left\|\ell(t) \rho_{0} \bar{v}_{t t}^{\prime \prime}(t) \bar{v}^{\prime}(t)\right\|_{0} \leq C_{M}$.

In particular, using (5.19), we see that

$$
\frac{1}{\rho_{0}}\left(\rho_{0} Z\right)^{\prime}=\rho_{0} \partial_{t}^{4} \check{v}^{\prime \prime}+2 \rho_{0}^{\prime} \partial_{t}^{4} \check{v}^{\prime}
$$

so that

$$
\begin{aligned}
\kappa\left\|\rho_{0} \partial_{t}^{4} \check{v}^{\prime \prime \prime}+3 \rho_{0}^{\prime} \partial_{t}^{4} \check{v}^{\prime \prime}+2 \rho_{0}^{\prime \prime} \partial_{t}^{4} \check{v}^{\prime}\right\|_{0} \leq \\
C_{M}+\left\|\rho_{0} \bar{v}_{t t t}^{\prime \prime \prime}(t)\right\|_{0}+\left\|\bar{v}_{t t t}^{\prime \prime}(t)\right\|_{0}+C\|Y\|_{1}+\mathcal{N}_{0},
\end{aligned}
$$

which implies that

$$
\begin{aligned}
\kappa \| & \left(\rho_{0} \partial_{t}^{4} \check{v}\right)^{\prime \prime \prime} \|_{0} \\
\leq & C_{M}+\left\|\rho_{0} \bar{v}_{t t t}^{\prime \prime \prime}(t)\right\|_{0}+\left\|\bar{v}_{t t t}^{\prime \prime}(t)\right\|_{0}+C\|Y\|_{1}+\mathcal{N}_{0}+\kappa\left\|\rho_{0}^{\prime \prime \prime} \partial_{t}^{4} \check{v}\right\|_{0} \\
& +\kappa\left\|3 \rho_{0}^{\prime \prime} \partial_{t}^{4} \check{v}^{\prime}\right\|_{0} \\
\leq & C_{M}+\left\|\rho_{0} \bar{v}_{t t t}^{\prime \prime \prime}(t)\right\|_{0}+\left\|\bar{v}_{t t t}^{\prime \prime}(t)\right\|_{0}+C\|Y\|_{1}+\mathcal{N}_{0} \\
& +\kappa\left(\left\|\rho_{0}^{\prime \prime \prime}\right\|_{L^{\infty}}+3\left\|\rho_{0}^{\prime \prime}\right\|_{L^{\infty}}\right)\|Z\|_{1},
\end{aligned}
$$


where we have used (5.20) in the second inequality above. Having established in (5.20) that $\partial_{t}^{4} \breve{v} \in H^{1}(I),(5.15)$ together with (5.17) show that $\left(\rho_{0} \partial_{t}^{4} \breve{v}\right)^{\prime \prime},\left(\rho_{0} \partial_{t}^{4} \breve{v}\right)^{\prime}$, and $\rho_{0} \partial_{t}^{4} \breve{v}$ are bounded in $L^{2}(I)$ by the right-hand side of (5.21). Therefore,

$$
\begin{aligned}
\kappa\left\|\rho_{0} \partial_{t}^{4} \check{v}\right\|_{3} \leq & C_{M}+\left\|\rho_{0} \bar{v}_{t t t}^{\prime \prime \prime}(t)\right\|_{0}+\left\|\bar{v}_{t t t}^{\prime \prime}(t)\right\|_{0}+C\|Y\|_{1}+\mathcal{N}_{0} \\
& +\kappa\left(\left\|\rho_{0}^{\prime \prime \prime}\right\|_{L^{\infty}}+3\left\|\rho_{0}^{\prime \prime}\right\|_{L^{\infty}}\right)\|Z\|_{1} .
\end{aligned}
$$

Now, thanks to our high-order Hardy-type inequality, we infer from (5.22) that

$$
\begin{aligned}
\kappa\left\|\partial_{t}^{4} \check{v}\right\|_{2} \leq & C_{M}+\left\|\rho_{0} \bar{v}_{t t t}^{\prime \prime \prime}(t)\right\|_{0}+\left\|\bar{v}_{t t t}^{\prime \prime}(t)\right\|_{0}+C\|Y\|_{1}+\mathcal{N}_{0} \\
& +\kappa\left(\left\|\rho_{0}^{\prime \prime \prime}\right\|_{L^{\infty}}+3\left\|\rho_{0}^{\prime \prime}\right\|_{L^{\infty}}\right)\|Z\|_{1} .
\end{aligned}
$$

Next we see that (5.22) implies that

$$
\begin{aligned}
\kappa\left\|\rho_{0} \partial_{t}^{4} \check{v}^{\prime}+\rho^{\prime} \partial_{t}^{4} \check{v}\right\|_{2} \leq & C_{M}+\left\|\rho_{0} \bar{v}_{t t t}^{\prime \prime \prime}(t)\right\|_{0}+\left\|\bar{v}_{t t t}^{\prime \prime}(t)\right\|_{0}+C\|Y\|_{1}+\mathcal{N}_{0} \\
& +\kappa\left(\left\|\rho_{0}^{\prime \prime \prime}\right\|_{L^{\infty}}+3\left\|\rho_{0}^{\prime \prime}\right\|_{L^{\infty}}\right)\|Z\|_{1},
\end{aligned}
$$

which thanks to (5.23) and (5.19) implies that

$$
\begin{aligned}
\kappa\|Z\|_{2} \leq & C_{M}+\left\|\rho_{0} \bar{v}_{t t t}^{\prime \prime \prime}(t)\right\|_{0}+\left\|\bar{v}_{t t t}^{\prime \prime}(t)\right\|_{0}+C\|Y\|_{1}+\mathcal{N}_{0} \\
& +\kappa\left(\left\|\rho_{0}^{\prime \prime \prime}\right\|_{L^{\infty}}+3\left\|\rho_{0}^{\prime \prime}\right\|_{L^{\infty}}\right)\|Z\|_{1} .
\end{aligned}
$$

\subsection{Definition of $v$ and Existence of a Fixed Point}

We are now in a position to define $v$ in the following fashion: let us first define on $[0, T]$

$$
f(t)=u_{0}(0)-\int_{0}^{t} \frac{1}{\rho_{0}}\left[\frac{\rho_{0}^{2}}{\bar{\eta}^{\prime 2}}\right]^{\prime}(\cdot, 0)+\kappa \int_{0}^{t} \frac{1}{\rho_{0}}\left[\rho_{0} X\right]^{\prime}(\cdot, 0),
$$

which is well-defined thanks to (5.24) and (5.15). We next define

$$
v(t, x)=f(t)+\check{v}(t, x) .
$$

We then notice that from (5.8), we immediately have that

$$
v_{t}^{\prime}+\left[\frac{1}{\rho_{0}}\left[\frac{\rho_{0}^{2}}{\bar{\eta}^{\prime 2}}\right]^{\prime}\right]^{\prime}-\kappa\left[\frac{1}{\rho_{0}}\left[\rho_{0}^{2} v^{\prime}\right]^{\prime}\right]^{\prime}=0,
$$

from which we infer that in $[0, T] \times I$

$$
v_{t}+\frac{1}{\rho_{0}}\left[\frac{\rho_{0}^{2}}{\bar{\eta}^{\prime 2}}\right]^{\prime}-\kappa \frac{1}{\rho_{0}}\left[\rho_{0}^{2} v^{\prime}\right]^{\prime}=g(t)
$$

for some function $g$ depending only on $t$. By taking the trace of this equation on the left endpoint $x=0$, we see that

$$
v_{t}(t, 0)+\frac{1}{\rho_{0}}\left[\frac{\rho_{0}^{2}}{\bar{\eta}^{\prime 2}}\right]^{\prime}(t, 0)-\kappa \frac{1}{\rho_{0}}\left[\rho_{0}^{2} v^{\prime}\right]^{\prime}(t, 0)=g(t),
$$

which together with the identity

$$
v_{t}(t, 0)=f_{t}(t)=-\frac{1}{\rho_{0}}\left[\frac{\rho_{0}^{2}}{\bar{\eta}^{\prime 2}}\right]^{\prime}(t, 0)+\kappa \frac{1}{\rho_{0}}\left[\rho_{0}^{2} v^{\prime}\right]^{\prime}(t, 0)
$$


shows that $g(t)=0$. Therefore, $v$ is a solution of (5.7), and also satisfies by construction $v(0, \cdot)=u_{0}(\cdot)$.

We can now establish the existence of a fixed point for the mapping $\bar{v} \rightarrow v$ in $\mathcal{C}_{T}(M)$, with $T$ taken sufficiently small and depending a priori on $\kappa$. We first notice that, thanks to the estimates (5.24) and (5.15), we have the inequality

$$
\left\|\partial_{t}^{4} f(t)\right\|_{L^{2}(0, T)} \leq \mathcal{N}_{0}+C_{M},
$$

which together with (5.23) and (5.15) provides the estimate

$$
\left\|\partial_{t}^{4} v\right\|_{L^{2}\left(0, T ; H^{2}(I)\right)} \leq C \mathcal{N}_{0}+C_{K} C_{M}
$$

Then, (5.22) implies that

$$
\left\|\rho_{0} \partial_{t}^{4} v\right\|_{L^{2}\left(0, T ; H^{3}(I)\right)}+\left\|\partial_{t}^{4} v\right\|_{L^{2}\left(0, T ; H^{2}(I)\right)} \leq C \mathcal{N}_{0}+C_{K} C_{M} .
$$

Using equation (5.7), we then see that

$$
\left\|\partial_{t}^{5} v\right\|_{L^{2}\left(0, T ; L^{2}(I)\right)} \leq C \mathcal{N}_{0}+C_{K} C_{M}
$$

and combining this with (5.16) shows that

$$
\left\|\partial_{t}^{5} v\right\|_{L^{2}\left(0, T ; H^{1}(I)\right)}+\left\|\rho_{0} \partial_{t}^{5} v^{\prime}\right\|_{L^{2}\left(0, T ; H^{1}(I)\right)} \leq C \mathcal{N}_{0}+C_{K} C_{M} .
$$

The fundamental theorem of calculus applied to (5.27) and (5.28) then shows that

$$
\begin{aligned}
& \left\|\rho_{0} \partial_{t}^{3} v\right\|_{L^{2}\left(0, T ; H^{3}(I)\right)}+\left\|\partial_{t}^{3} v\right\|_{L^{2}\left(0, T ; H^{2}(I)\right)} \\
& +\left\|\partial_{t}^{4} v\right\|_{L^{2}\left(0, T ; H^{1}(I)\right)}+\left\|\rho_{0} \partial_{t}^{4} v^{\prime}\right\|_{L^{2}\left(0, T ; H^{1}(I)\right)} \\
& \quad \leq \mathcal{N}_{0}+\sqrt{T}\left(C \mathcal{N}_{0}+C_{K} C_{M}\right) .
\end{aligned}
$$

In turn, (5.29) shows that for

$$
T \leq \frac{1}{\left(C \mathcal{N}_{0}+C_{\kappa} C_{M}\right)^{2}}
$$

$v \in \mathcal{C}_{T}(M)$. It is also clear that there is only one solution $v \in L^{2}\left(0, T ; H^{2}(I)\right)$ of (5.7) with $v(0)=u_{0}$ (where this initial condition is well-defined due to the fact that $\left.\left\|v_{t}\right\|_{L^{2}\left(0, T ; H^{1}(I)\right)} \leq \mathcal{N}_{0}+C_{\kappa} \sqrt{T} C_{M}\right)$, since if we denote by $w$ another solution with the same regularity, the difference $\delta v=v-w$ satisfies $\delta v(0, \cdot)=0$ with $\rho_{0} \delta v_{t}-\kappa\left[\rho_{0}^{2} \delta v^{\prime}\right]^{\prime}=0$, which implies

$$
\frac{1}{2} \frac{d}{d t} \int_{I} \rho_{0} \delta v^{2}+\kappa \int_{I} \rho_{0}^{2} \delta v^{2}=0,
$$

which with $\delta v(0, \cdot)=0$ implies $\delta v=0$. So the mapping $\bar{v} \rightarrow v$ is well-defined and, thanks to (5.26), is a mapping from $\mathcal{C}_{T}(M)$ into itself for $T=T_{\kappa}$ satisfying inequality (5.30)).

Because it is furthermore clear that, in the $L^{2}\left(0, T_{\kappa} ; H^{2}(I)\right)$ norm, the mapping is weakly continuous, the Tychonoff fixed-point theorem [5] provides us with the existence of a fixed point to this mapping. Such a fixed point, which we denote by 
$v_{\kappa}$, is a solution of the nonlinear degenerate parabolic $\kappa$-problem (4.5), with initial condition $v_{\kappa}(0, \cdot)=u_{0}(\cdot)$. It should be clear that the fixed point $v_{\kappa}$ also satisfies (5.16) and (5.27) so that

$$
\begin{aligned}
& \left\|\rho_{0} \partial_{t}^{4} v_{\kappa}\right\|_{L^{2}\left(0, T ; H^{3}(I)\right)}+\left\|\partial_{t}^{4} v_{\kappa}\right\|_{L^{2}\left(0, T ; H^{2}(I)\right)} \\
& \quad+\left\|\rho_{0} \partial_{t}^{5} v_{\kappa}^{\prime}\right\|_{L^{2}\left(0, T ; H^{1}(I)\right)}+\left\|\partial_{t}^{5} v_{\kappa}\right\|_{L^{2}\left(0, T ; H^{1}(I)\right)} \leq C,
\end{aligned}
$$

In the next section, we establish for $v_{\kappa}$ in $L^{2}\left(0, T_{\kappa} ; H^{2}(I)\right) \kappa$-independent estimates (which are indeed possible because our parabolic approximate $\kappa$-problem respects the structure of the original compressible Euler equations (1.11)), from which we infer a short time interval of existence [0,T], with $T$ independent of $\kappa$. These $\kappa$-independent estimates will allow us to pass to the weak limit of the sequence $v_{\kappa}$ as $\kappa \rightarrow 0$ to obtain the solution to (1.11).

\section{Asymptotic Estimates for $v_{\kappa}$ That Are Independent of $\kappa$}

\subsection{Higher-Order Energy Function Appropriate for Asymptotic Estimates as $\boldsymbol{\kappa} \rightarrow \mathbf{0}$}

Our objective in this section is to show that the higher-order energy function $E$ defined in (1.14) satisfies the inequality

$$
\sup _{t \in[0, T]} E(t) \leq M_{0}+C T P\left(\sup _{t \in[0, T]} E(t)\right),
$$

where $P$ denotes a generic polynomial function of its argument, for $T>0$ taken sufficiently small, with $M_{0}$ defined in (1.14). The norms in $E(t):=E\left(t, v_{\kappa}\right)$ are for solutions $v_{\kappa}$ to our degenerate parabolic $\kappa$-problem (4.5). According to Theorem 5.1, $v_{\kappa} \in X_{T_{\kappa}}$ with the additional bound $\left\|\partial_{t}^{4} v_{\kappa}\right\|_{L^{2}\left(0, T_{\kappa} ; H^{2}(I)\right)}<\infty$ provided by (5.26). As such, the energy function $E$ is continuous with respect to $t$, and inequality (6.1) would thus establish a time interval of existence and bound that are both independent of $\kappa$. For the sake of notational convenience, we shall denote $v_{\kappa}$ by $\tilde{v}$.

\subsection{A $\kappa$-Independent Energy Estimate on the Fifth Time-Differentiated Problem}

Our starting point shall be the fifth time-differentiated problem of (4.5) for which we have, by naturally using $\partial_{t}^{5} \tilde{v} \in L^{2}\left(0, T_{\kappa} ; H^{1}(I)\right)$ as a test function, the following identity:

$$
\underbrace{\frac{1}{2} \frac{d}{d t} \int_{I} \rho_{0}\left|\partial_{t}^{5} \tilde{v}\right|^{2}}_{\mathcal{I}_{1}}-\underbrace{\int_{I} \partial_{t}^{5}\left[\frac{\rho_{0}^{2}}{\tilde{\eta}^{\prime 2}}\right] \partial_{t}^{5} \tilde{v}^{\prime}}_{\mathcal{I}_{2}}+\underbrace{\kappa \int_{I} \rho_{0}^{2}\left(\partial_{t}^{5} \tilde{v}^{\prime}\right)^{2}=0}_{\mathcal{I}_{3}} .
$$

In order to form the exact time derivative in the term $\mathcal{I}_{1}$, we rely on the fact that the solutions we constructed to (4.5) satisfy $\partial_{t}^{6} v \in L^{2}\left(0, T_{\kappa} ; L^{2}(I)\right)$, which follows 
from the relation

$$
\partial_{t}^{6} \tilde{v}=\partial_{t}^{5}\left[\frac{2}{\tilde{\eta}^{\prime}}\left(\frac{\rho_{0}}{\tilde{\eta}^{\prime}}\right)^{\prime}\right]+\frac{\kappa}{\rho_{0}}\left[\rho_{0}^{2} \partial_{t}^{5} v^{\prime}\right]^{\prime},
$$

and estimate (5.31). Upon integration in time, both the terms $\mathcal{I}_{1}$ and $\mathcal{I}_{3}$ provide sign-definite energy contributions, so we focus our attention on the nonlinear estimates required of the term $\mathcal{I}_{2}$.

We see that

$$
\begin{aligned}
-\mathcal{I}_{2} & =2 \int_{I} \partial_{t}^{4} \tilde{v}^{\prime}\left[\frac{\rho_{0}^{2}}{\tilde{\eta}^{\prime 3}}\right] \partial_{t}^{5} \tilde{v}^{\prime}+\sum_{a=1}^{4} c_{a} \int_{I} \partial_{t}^{5-a} \frac{1}{\tilde{\eta}^{\prime}} \partial_{t}^{a} \frac{1}{\tilde{\eta}^{\prime}} \rho_{0}^{2} \partial_{t}^{5} \tilde{v}^{\prime} \\
& =\frac{d}{d t} \int_{I}\left(\partial_{t}^{4} \tilde{v}^{\prime}\right)^{2} \frac{\rho_{0}^{2}}{\tilde{\eta}^{\prime 3}}+3 \int_{I}\left(\partial_{t}^{4} \tilde{v}^{\prime}\right)^{2} \tilde{v}^{\prime} \frac{\rho_{0}^{2}}{\tilde{\eta}^{\prime 4}}+\sum_{a=1}^{4} c_{a} \int_{I} \partial_{t}^{5-a} \frac{1}{\tilde{\eta}^{\prime}} \partial_{t}^{a} \frac{1}{\tilde{\eta}^{\prime}} \rho_{0}^{2} \partial_{t}^{5} \tilde{v}^{\prime} .
\end{aligned}
$$

Hence integrating (6.2) from 0 to $t \in\left[0, T_{\kappa}\right]$, we find that

$$
\begin{aligned}
\frac{1}{2} \int_{I} \rho_{0} \partial_{t}^{5} \tilde{v}^{2}(t)+\int_{I}\left(\partial_{t}^{4} \tilde{v}^{\prime}\right)^{2} \frac{\rho_{0}^{2}}{\tilde{\eta}^{\prime 3}}(t)+\kappa \int_{0}^{t} \int_{I} \rho_{0}^{2}\left(\partial_{t}^{5} \tilde{v}^{\prime}\right)^{2} \\
=\frac{1}{2} \int_{I} \rho_{0} \partial_{t}^{5} \tilde{v}^{2}(0)+\int_{I}\left(\partial_{t}^{4} \tilde{v}^{\prime}\right)^{2} \frac{\rho_{0}^{2}}{\tilde{\eta}^{\prime 3}}(0)-3 \int_{0}^{t} \int_{I}\left(\partial_{t}^{4} \tilde{v}^{\prime}\right)^{2} \tilde{v}^{\prime} \frac{\rho_{0}^{2}}{\tilde{\eta}^{\prime 4}} \\
\quad-\sum_{a=1}^{4} c_{a} \int_{0}^{t} \int_{I} \partial_{t}^{5-a} \frac{1}{\tilde{\eta}^{\prime}} \partial_{t}^{a} \frac{1}{\tilde{\eta}^{\prime}} \rho_{0}^{2} \partial_{t}^{5} \tilde{v}^{\prime}
\end{aligned}
$$

We next show that all of the error terms, comprising the right-hand side of (6.3), can be bounded by $C t P\left(\sup _{[0, t]} E\right)$. For the first space-time integral appearing on the right-hand side of (6.3), it is clear that

$$
-3 \int_{0}^{t} \int_{I}\left(\partial_{t}^{4} \tilde{v}^{\prime}\right)^{2} \tilde{v}^{\prime} \frac{\rho_{0}^{2}}{\tilde{\eta}^{\prime 4}} \leq C t P\left(\sup _{[0, t]} E\right) .
$$

We now study the last integrals on the right-hand side of (6.3). Using integration by parts in time, we have that

$$
\begin{aligned}
& \int_{0}^{t} \int_{I} \partial_{t}^{5-a} \frac{1}{\tilde{\eta}^{\prime}} \partial_{t}^{a} \frac{1}{\tilde{\eta}^{\prime}} \rho_{0}^{2} \partial_{t}^{5} \tilde{v}^{\prime}= \\
& \quad-\int_{0}^{t} \int_{I}\left[\partial_{t}^{5-a} \frac{1}{\tilde{\eta}^{\prime}} \partial_{t}^{a} \frac{1}{\tilde{\eta}^{\prime}}\right]_{t} \rho_{0}^{2} \partial_{t}^{4} \tilde{v}^{\prime}+\left.\int_{I} \partial_{t}^{5-a} \frac{1}{\tilde{\eta}^{\prime}} \partial_{t}^{a} \frac{1}{\tilde{\eta}^{\prime}} \rho_{0}^{2} \partial_{t}^{4} \tilde{v}^{\prime}\right|_{0} ^{t} .
\end{aligned}
$$

We first consider the space-time integral on the right-hand side of (6.5). As the sum is taken for $a=1$ to 4 , we then see that it will be written under the form of 
the sum of space-time integrals of the following type:

$$
\begin{aligned}
& I_{1}=\int_{0}^{t} \int_{I} \rho_{0} \partial_{t}^{4} \tilde{v}^{\prime} R(\tilde{\eta}) \rho_{0} \partial_{t}^{4} \tilde{v}^{\prime}, \\
& I_{2}=\int_{0}^{t} \int_{I} \rho_{0} \partial_{t}^{3} \tilde{v}^{\prime} v^{\prime} \partial_{t} \tilde{v}^{\prime} R(\tilde{\eta}) \rho_{0} \partial_{t}^{4} \tilde{v}^{\prime}, \\
& I_{3}=\int_{0}^{t} \int_{I} \rho_{0} \partial_{t}^{2} \tilde{v}^{\prime} v^{\prime} \partial_{t}^{2} \tilde{v}^{\prime} R(\tilde{\eta}) \rho_{0} \partial_{t}^{4} \tilde{v}^{\prime}, \\
& I_{4}=\int_{0}^{t} \int_{I} \rho_{0} \partial_{t}^{2} \tilde{v}^{\prime} v^{\prime} R(\tilde{\eta})\left(\partial_{t} \tilde{v}^{\prime}\right)^{2} \rho_{0} \partial_{t}^{4} \tilde{v}^{\prime},
\end{aligned}
$$

where $R(\tilde{\eta})$ denotes a rational function of $\tilde{\eta}^{\prime}$. We immediately see that

$$
\left|I_{1}\right| \leq C t P\left(\sup _{[0, t]} E\right) .
$$

Next, we have that

$$
\begin{aligned}
\left|I_{2}\right| & \leq C \int_{0}^{t} \int_{I}\left\|\rho_{0} \partial_{t}^{3} \tilde{v}^{\prime}\right\|_{L^{4}}\left\|v^{\prime}\right\|_{L^{\infty}}\left\|\partial_{t} \tilde{v}^{\prime}\right\|_{L^{4}}\|R(\tilde{\eta})\|_{L^{\infty}}\left\|\rho_{0} \partial_{t}^{4} \tilde{v}^{\prime}\right\|_{0} \\
& \leq C \int_{0}^{t} \int_{I}\left\|\rho_{0} \partial_{t}^{3} \tilde{v}^{\prime}\right\|_{H^{\frac{1}{2}}}\left\|v^{\prime}\right\|_{L^{\infty}}\left\|\partial_{t} \tilde{v}^{\prime}\right\|_{H^{1 / 2}}\|R(\tilde{\eta})\|_{L^{\infty}}\left\|\rho_{0} \partial_{t}^{4} \tilde{v}^{\prime}\right\|_{0} \\
& \leq C t P\left(\sup _{[0, t]} E\right) .
\end{aligned}
$$

Similarly,

$$
\begin{aligned}
\left|I_{3}\right| & \leq C \int_{0}^{t}\left\|\rho_{0} \partial_{t}^{2} \tilde{v}^{\prime}\right\|_{L^{\infty}}\left\|v^{\prime}\right\|_{L^{\infty}}\left\|\partial_{t}^{2} \tilde{v}^{\prime}\right\|_{0}\|R(\tilde{\eta})\|_{L^{\infty}}\left\|\rho_{0} \partial_{t}^{4} \tilde{v}^{\prime}\right\|_{0} \\
& \leq C t P\left(\sup _{[0, t]} E\right),
\end{aligned}
$$

and

$$
\begin{aligned}
\left|I_{4}\right| & \leq C \int_{0}^{t}\left\|\rho_{0} \partial_{t}^{2} \tilde{v}^{\prime}\right\|_{L^{\infty}}\left\|v^{\prime}\right\|_{L^{\infty}}\left\|\partial_{t} \tilde{v}^{\prime}\right\|_{L^{4}}^{2}\|R(\tilde{\eta})\|_{L^{\infty}}\left\|\rho_{0} \partial_{t}^{4} \tilde{v}^{\prime}\right\|_{0} \\
& \leq C t P\left(\sup _{[0, t]} E\right),
\end{aligned}
$$

where we have used the fact that in one dimension, $\|\cdot\|_{L^{\infty}} \leq C\|\cdot\|_{H^{1}}$ and $\|\cdot\|_{L^{4}} \leq C\|\cdot\|_{H^{1 / 2}}$. 
Next, we treat the second term on the right-hand side of (6.5), beginning with the case $a=1$. We see that for $\delta>0$

$$
\begin{aligned}
\left.\int_{I} \partial_{t}^{4} \frac{1}{\tilde{\eta}^{\prime}} \partial_{t} \frac{1}{\tilde{\eta}^{\prime}} \rho_{0}^{2} \partial_{t}^{4} \tilde{v}^{\prime}\right|_{0} ^{t}= & \int_{I}\left(R(\tilde{\eta}) \partial_{t}^{3} \tilde{v}^{\prime} \tilde{v}^{\prime} \rho_{0}^{2} \partial_{t}^{4} \tilde{v}^{\prime}\right)(t) \\
& -\int_{I}\left(R(\tilde{\eta}) \partial_{t}^{3} \tilde{v}^{\prime} \tilde{v}^{\prime} \rho_{0}^{2} \partial_{t}^{4} \tilde{v}^{\prime}\right)(0),
\end{aligned}
$$

and

$$
\begin{aligned}
\int_{I} & \left(R(\tilde{\eta}) \partial_{t}^{3} \tilde{v}^{\prime} \tilde{v}^{\prime} \rho_{0}^{2} \partial_{t}^{4} \tilde{v}^{\prime}\right)(t) \\
= & \int_{I}\left[\rho_{0} \partial_{t}^{3} \tilde{v}^{\prime}(0)+\int_{0}^{t} \rho_{0} \partial_{t}^{4} \tilde{v}^{\prime}\right] R(\tilde{\eta}(t)) \tilde{v}^{\prime}(t) \rho_{0} \partial_{t}^{4} \tilde{v}^{\prime}(t) \\
\leq & \underset{[0, T]}{\left.C \sup _{[0, T]} E\right)+\int_{I} \rho_{0} \partial_{t}^{3} \tilde{v}^{\prime}(0) R(\tilde{\eta}(t)) \tilde{v}^{\prime}(t) \rho_{0} \partial_{t}^{4} \tilde{v}^{\prime}(t)} \\
\leq & C t P\left(\sup _{[0, T]} E\right)+\delta\left\|\rho_{0} \partial_{t}^{4} \tilde{v}^{\prime}(t)\right\|_{0}^{2}+C_{\delta}\left\|\rho_{0} \partial_{t}^{3} \tilde{v}^{\prime}(0) R(\tilde{\eta}(t))\right\|_{L^{4}(I)}^{2}\left\|\tilde{v}^{\prime}(t)\right\|_{L^{4}(I)}^{2} \\
\leq & C t P\left(\sup _{[0, T]} E\right)+\delta\left\|\rho_{0} \partial_{t}^{4} \tilde{v}^{\prime}(t)\right\|_{0}^{2} \\
& +C_{\delta}\left\|\rho_{0} \partial_{t}^{3} \tilde{v}^{\prime}(0) R(\tilde{\eta}(t))\right\|_{L^{4}(I)}^{2}\left\|\tilde{v}^{\prime}(0)+\int_{0}^{t} \tilde{v}_{t}^{\prime}\right\|_{L^{4}(I)}^{2} \\
\leq & M_{0}+C t P\left(\sup _{[0, T]} E\right)+\delta\left\|\rho_{0} \partial_{t}^{4} \tilde{v}^{\prime}(t)\right\|_{0}^{2},
\end{aligned}
$$

where the last inequality follows from the fact that

$$
\|R(\tilde{\eta})\|_{L^{\infty}(I)}^{2} \leq M_{0}+C t P\left(\sup _{[0, T]} E\right),
$$

which itself follows from the fundamental theorem of calculus. The case when $a=$ 2,3, 4 can be estimated in the same fashion; hence, by taking $\delta>0$ sufficiently small so that $\delta\left\|\rho_{0} \partial_{t}^{4} \tilde{v}^{\prime}(t)\right\|_{0}^{2}$ is absorbed by the left-hand side of (6.3), we obtain the desired inequality. In particular, putting together estimates (6.3)-(6.7) with the inequality directly above provides us with

$$
\frac{1}{2} \int_{I} \rho_{0} \partial_{t}^{5} \tilde{v}^{2}(t)+\int_{I}\left(\partial_{t}^{4} \tilde{v}^{\prime}\right)^{2} \frac{\rho_{0}^{2}}{\tilde{\eta}^{\prime 3}}(t)+\kappa \int_{0}^{t} \int_{I} \rho_{0}^{2}\left(\partial_{t}^{5} \tilde{v}^{\prime}\right)^{2} \leq M_{0}+C t P\left(\sup _{[0, t]} E\right),
$$


and thus, employing the fundamental theorem of calculus,

$$
\begin{aligned}
& \frac{1}{2} \int_{I} \rho_{0} \partial_{t}^{5} \tilde{v}^{2}(t)+\int_{I}\left(\rho_{0} \partial_{t}^{4} \tilde{v}^{\prime}\right)^{2}(t)+\kappa \int_{0}^{t} \int_{I} \rho_{0}^{2}\left(\partial_{t}^{5} \tilde{v}^{\prime}\right)^{2} \\
& \quad \leq M_{0}+C t P\left(\sup _{[0, t]} E\right)+3 \int_{I}\left(\partial_{t}^{4} \tilde{v}^{\prime}\right)^{2}(t) \rho_{0}^{2} \int_{0}^{t} \frac{\tilde{v}^{\prime}}{\tilde{\eta}^{\prime 4}} \\
& \quad \leq M_{0}+C t P\left(\sup _{[0, t]} E\right) .
\end{aligned}
$$

Note that although our definition of $M_{0}$ lacks the term $\left\|\sqrt{\rho_{0}} \partial_{t}^{5} v(0)\right\|_{0}^{2}$, it is implicitly bounded by $M_{0}$ using equation (5.1) with $k=5$, as well as estimate (5.6) for the highest-order term that has the $\kappa$-coefficient.

\subsection{Elliptic and Hardy-Type Estimates for $\partial_{t}^{2} v(t)$}

Having obtained the energy estimate (6.10) for the fifth time-differentiated problem, we can begin our bootstrapping argument. We now consider the third timedifferentiated version of (4.5a),

$$
\left[\partial_{t}^{3} \frac{\rho_{0}^{2}}{\tilde{\eta}^{\prime 2}}\right]^{\prime}-\kappa\left[\rho_{0}^{2} \partial_{t}^{3} \tilde{v}^{\prime}\right]^{\prime}=-\rho_{0} \partial_{t}^{4} \tilde{v},
$$

which can be written as

$$
-2\left[\frac{\rho_{0}^{2} \partial_{t}^{2} \tilde{v}^{\prime}}{\tilde{\eta}^{\prime 3}}\right]^{\prime}-\kappa\left[\rho_{0}^{2} \partial_{t}^{3} \tilde{v}^{\prime}\right]^{\prime}=-\rho_{0} \partial_{t}^{4} \tilde{v}+c_{1}\left[\frac{\rho_{0}^{2} \partial_{t} \tilde{v}^{\prime} \tilde{v}^{\prime}}{\tilde{\eta}^{\prime 4}}\right]^{\prime}+c_{2}\left[\frac{\rho_{0}^{2} \tilde{v}^{\prime 3}}{\tilde{\eta}^{\prime 5}}\right]^{\prime},
$$

and finally rewritten as the following identity:

$$
\begin{aligned}
-2\left[\rho_{0}^{2} \partial_{t}^{2} \tilde{v}^{\prime}\right]^{\prime}-\kappa\left[\rho_{0}^{2} \partial_{t}^{3} \tilde{v}^{\prime}\right]^{\prime}= & -\rho_{0} \partial_{t}^{4} \tilde{v}+c_{1}\left[\frac{\rho_{0}^{2} \partial_{t} \tilde{v}^{\prime} \tilde{v}^{\prime}}{\tilde{\eta}^{\prime 4}}\right]^{\prime}+c_{2}\left[\frac{\rho_{0}^{2} \tilde{v}^{\prime 3}}{\tilde{\eta}^{\prime 5}}\right]^{\prime} \\
& -2\left[\rho_{0}^{2} \partial_{t}^{2} \tilde{v}^{\prime}\right]^{\prime}\left(1-\frac{1}{\tilde{\eta}^{\prime 3}}\right)-6 \rho_{0}^{2} \partial_{t}^{2} \tilde{v}^{\prime} \frac{\tilde{\eta}^{\prime \prime}}{\tilde{\eta}^{\prime 4}}
\end{aligned}
$$

Here $c_{1}$ and $c_{2}$ are constants whose exact value is not important.

Therefore, using Lemma 3.2 together with the fundamental theorem of calculus for the fourth term on the right-hand side of (6.11), we obtain that for any $t \in$ $\left[0, T_{\kappa}\right]$,

$$
\begin{aligned}
\sup _{[0, t]} \| & \frac{2}{\rho_{0}}\left[\rho_{0}^{2} \partial_{t}^{2} \tilde{v}^{\prime}\right]^{\prime} \|_{0} \\
\leq & \sup _{[0, t]}\left\|\partial_{t}^{4} \tilde{v}\right\|_{0}+\sup _{[0, t]}\left\|\frac{c_{1}}{\rho_{0}}\left[\frac{\rho_{0}^{2} \partial_{t} \tilde{v}^{\prime} \tilde{v}^{\prime}}{\tilde{\eta}^{\prime 4}}\right]^{\prime}\right\|_{0}+\sup _{[0, t]}\left\|\frac{c_{2}}{\rho_{0}}\left[\frac{\rho_{0}^{2} \tilde{v}^{\prime 3}}{\tilde{\eta}^{\prime 5}}\right]^{\prime}\right\|_{0} \\
& +\sup _{[0, t]}\left\|\frac{2}{\rho_{0}}\left[\rho_{0}^{2} \partial_{t}^{2} \tilde{v}^{\prime}\right]^{\prime}\right\|_{0}\left\|3 \int_{0}^{\cdot} \frac{\tilde{v}^{\prime}}{\tilde{\eta}^{\prime 4}}\right\|_{L^{\infty}}+6 \sup _{[0, t]}\left\|\rho_{0} \partial_{t}^{2} \tilde{v}^{\prime} \frac{\tilde{\eta}^{\prime \prime}}{\tilde{\eta}^{\prime 4}}\right\|_{0} .
\end{aligned}
$$


We next estimate each term on the right-hand side of (6.12). For the first term, we will use our estimate (6.10), from which we infer for each $t \in\left[0, T_{\kappa}\right]$

$$
\int_{I} \rho_{0}^{2}\left[\partial_{t}^{4} \tilde{v}^{2}+\partial_{t}^{4} \tilde{v}^{\prime 2}\right](t) \leq M_{0}+C t P\left(\sup _{[0, t]} E\right) .
$$

Note that the first term on the left-hand side of (6.13) comes from the first term of (6.10), together with the fact that $\partial_{t}^{4} v(t, x)=v_{4}(x)+\int_{0}^{t} \partial_{t}^{5} v(\cdot, x)$. Therefore, the Sobolev weighted embedding estimate (2.1) provides us with the following estimate:

$$
\int_{I} \partial_{t}^{4} \tilde{v}^{2}(t) \leq M_{0}+C t P\left(\sup _{[0, t]} E\right) .
$$

The remaining terms will be estimated by simply using the definition of the energy function $E$. For the second term on the right-hand side of (6.12), we have that

$$
\begin{aligned}
& \left\|\frac{1}{\rho_{0}}\left[\frac{\rho_{0}^{2} \partial_{t} \tilde{v}^{\prime} \tilde{v}^{\prime}}{\tilde{\eta}^{\prime 4}}\right]^{\prime}\right\|_{0} \\
& \leq\left\|\left(\rho_{0} v_{t}^{\prime}\right)^{\prime}\right\|_{0}\left\|\frac{\tilde{v}^{\prime}}{\tilde{\eta}^{\prime 4}}\right\|_{L^{\infty}}+\left\|\tilde{v}_{t}^{\prime}\left[\frac{\rho_{0} \tilde{v}^{\prime}}{\tilde{\eta}^{\prime 4}}\right]^{\prime}\right\|_{0} \\
& \leq C\left\|\left(\rho_{0} \tilde{v}_{t}^{\prime}\right)^{\prime}\right\|_{0}\left\|\tilde{v}^{\prime}\right\|_{3 / 4}+\left\|\tilde{v}_{t}^{\prime}\left[\frac{\rho_{0}^{\prime} \tilde{v}^{\prime}}{\tilde{\eta}^{\prime 4}}\right]\right\|_{0}+\left\|\tilde{v}_{t}^{\prime}\left[\frac{\rho_{0} \tilde{v}^{\prime \prime}}{\tilde{\eta}^{\prime 4}}\right]\right\|_{0} \\
& +4\left\|\tilde{v}_{t}^{\prime}\left[\frac{\rho_{0} \tilde{v}^{\prime} \tilde{\eta}^{\prime \prime}}{\tilde{\eta}^{\prime 5}}\right]\right\|_{0} \\
& \leq C\left\|\left(\rho_{0} v_{1}^{\prime}\right)^{\prime}+\int_{0}^{\cdot}\left(\rho_{0} v_{t t}^{\prime}\right)^{\prime}\right\|_{0}\left\|\tilde{v}^{\prime}\right\|_{1}^{1 / 2}\left\|v_{1}^{\prime}+\int_{0}^{\cdot} \tilde{v}_{t}^{\prime}\right\|_{1 / 2}^{1 / 2} \\
& +C\left\|v_{1}^{\prime}+\int_{0}^{\cdot} \tilde{v}_{t t}^{\prime}\right\|_{0}\left\|\tilde{v}^{\prime}\right\|_{3 / 4}+C\left\|\tilde{v}_{t}^{\prime}\right\|_{0}\left\|\rho_{0} v^{\prime \prime}\right\|_{3 / 4} \\
& +C\left\|\tilde{v}^{\prime}\right\|_{3 / 4}\left\|\int_{0}^{\cdot} \tilde{v}^{\prime \prime}\right\|_{0}\left\|\rho_{0} v_{1}^{\prime}+\int_{0}^{\cdot} \rho_{0} \tilde{v}_{t t}^{\prime}\right\|_{1} \\
& \leq\left\|\left(\rho_{0} v_{1}^{\prime}\right)^{\prime}+\int_{0}^{\cdot}\left(\rho_{0} v_{t t}^{\prime}\right)^{\prime}\right\|_{0}\left\|\tilde{v}^{\prime}\right\|_{1}^{1 / 2}\left\|v_{1}^{\prime}+\int_{0} \tilde{v}_{t}^{\prime}\right\|_{1 / 2}^{1 / 2} \\
& +C\left\|v_{1}^{\prime}+\int_{0}^{\cdot} \tilde{v}_{t t}^{\prime}\right\|_{0}\left\|\tilde{v}^{\prime}\right\|_{1}^{1 / 2}\left\|v_{1}^{\prime}+\int_{0}^{\cdot} \tilde{v}_{t}^{\prime}\right\|_{1 / 2}^{1 / 2} \\
& +C\left\|\tilde{v}_{t}^{\prime}\right\|_{0}\left\|\rho_{0} v^{\prime \prime}\right\|_{1}^{3 / 4}\left\|\rho_{0} v_{0}^{\prime \prime}+\int_{0}^{\cdot} \rho_{0} v_{t}^{\prime \prime}\right\|_{0}^{1 / 4} \text {, }
\end{aligned}
$$


where we have used the fact that $\|\cdot\|_{L^{\infty}} \leq C\|\cdot\|_{3 / 4}$. Thanks to the definition of $E$, the previous inequality provides us, for any $t \in\left[0, T_{\kappa}\right]$, with

$$
\sup _{[0, t]}\left\|\frac{1}{\rho_{0}}\left[\frac{\rho_{0}^{2} \partial_{t} \tilde{v}^{\prime} \tilde{v}^{\prime}}{\tilde{\eta}^{\prime 4}}\right]^{\prime}\right\|_{0} \leq C \sup _{[0, t]} E^{3 / 4}\left(M_{0}+t P\left(\sup _{[0, t]} E\right)\right) .
$$

For the third term on the right-hand side of (6.12), we similarly have that

$$
\begin{aligned}
& \left\|\frac{1}{\rho_{0}}\left[\frac{\rho_{0}^{2} \tilde{v}^{\prime 3}}{\tilde{\eta}^{\prime 5}}\right]^{\prime}\right\|_{0} \\
& \leq\left\|\left(\rho_{0} v^{\prime}\right)^{\prime}\right\|_{L^{\infty}}\left\|\frac{\tilde{v}^{\prime 2}}{\tilde{\eta}^{\prime 5}}\right\|_{L^{2}}+\left\|\tilde{v}^{\prime}\left[\frac{\rho_{0} \tilde{v}^{\prime 2}}{\tilde{\eta}^{\prime 5}}\right]^{\prime}\right\|_{0} \\
& \leq C\left\|\left(\rho_{0} \tilde{v}^{\prime}\right)^{\prime}\right\|_{3 / 4}\left\|\tilde{v}^{\prime}\right\|_{1 / 2}^{2}+\left\|\tilde{v}^{\prime}\left[\frac{\rho_{0}^{\prime} \tilde{v}^{\prime 2}}{\tilde{\eta}^{\prime 5}}\right]\right\|_{0}+2\left\|\tilde{v}^{\prime}\left[\frac{\rho_{0} \tilde{v}^{\prime \prime} \tilde{v}^{\prime}}{\tilde{\eta}^{\prime 5}}\right]\right\|_{0} \\
& +5\left\|\tilde{v}^{\prime}\left[\frac{\rho_{0} \tilde{v}^{\prime 2} \tilde{\eta}^{\prime \prime}}{\tilde{\eta}^{\prime 6}}\right]\right\|_{0} \\
& \leq C\left\|\left(\rho_{0} v_{0}^{\prime}\right)^{\prime}+\int_{0}^{\cdot}\left(\rho_{0} v_{t}^{\prime}\right)^{\prime}\right\|_{0}^{1 / 4}\left\|\left(\rho_{0} \tilde{v}^{\prime}\right)^{\prime}\right\|_{1}^{3 / 4}\left\|\tilde{v}_{0}^{\prime}+\int_{0}^{\cdot} \tilde{v}_{t}^{\prime}\right\|_{1 / 2}^{2} \\
& +C\left\|\tilde{v}_{0}^{\prime}+\int_{0}^{\cdot} \tilde{v}_{t}^{\prime}\right\|_{1 / 2}^{3}+C\left\|\tilde{v}^{\prime}\right\|_{1 / 2}^{2}\left\|\rho_{0} v^{\prime \prime}\right\|_{L^{4}}+C\left\|\tilde{v}^{\prime}\right\|_{1 / 2}^{3}\left\|\rho_{0} \tilde{\eta}^{\prime \prime}\right\|_{L^{4}} \\
& \leq C\left\|\left(\rho_{0} v_{0}^{\prime}\right)^{\prime}+\int_{0}^{\cdot}\left(\rho_{0} v_{t}^{\prime}\right)^{\prime}\right\|_{0}^{1 / 4}\left\|\left(\rho_{0} \tilde{v}^{\prime}\right)^{\prime}\right\|_{1}^{3 / 4}\left\|\tilde{v}_{0}^{\prime}+\int_{0}^{\cdot} \tilde{v}_{t}^{\prime}\right\|_{1 / 2}^{2} \\
& +C\left\|\tilde{v}_{0}^{\prime}+\int_{0}^{\cdot} \tilde{v}_{t}^{\prime}\right\|_{1 / 2}^{3} \\
& +C\left\|\tilde{v}_{0}^{\prime}+\int_{0}^{\cdot} \tilde{v}_{t}^{\prime}\right\|_{1 / 2}^{2}\left\|\rho_{0} v_{0}^{\prime \prime}+\int_{0}^{\cdot} \rho_{0} v_{t}^{\prime \prime}\right\|_{0}^{1 / 2}\left\|\rho_{0} \tilde{v}^{\prime \prime}\right\|_{1}^{1 / 2} \\
& +C\left\|\tilde{v}_{0}^{\prime}+\int_{0}^{\cdot} \tilde{v}_{t}^{\prime}\right\|_{1 / 2}^{3}\left\|\int_{0}^{.} \rho_{0} v^{\prime \prime}\right\|_{1},
\end{aligned}
$$

where we have used the fact that $\|\cdot\|_{L^{p}} \leq C_{p}\|\cdot\|_{1 / 2}$ for all $1<p<\infty$. Again, using the definition of $E$, we obtain from the previous inequality for any $t \in\left[0, T_{\kappa}\right]$

$$
\sup _{[0, t]}\left\|\frac{1}{\rho_{0}}\left[\frac{\rho_{0}^{2} \tilde{v}^{\prime 3}}{\tilde{\eta}^{\prime 5}}\right]^{\prime}\right\|_{0} \leq C \sup _{[0, t]} E^{1 / 2}\left(M_{0}+t P\left(\sup _{[0, t]} E\right)\right) .
$$

For the fourth term of the right-hand side of (6.12), we see that

$$
\begin{aligned}
&\left\|\frac{2}{\rho_{0}}\left[\rho_{0}^{2} \partial_{t}^{2} \tilde{v}^{\prime}\right]^{\prime}\right\|_{0}\left\|3 \int_{0}^{\cdot} \frac{\tilde{v}^{\prime}}{\tilde{\eta}^{\prime 4}}\right\|_{L^{\infty}}(t) \leq \\
& C\left[\left\|\rho_{0} \partial_{t}^{2} v^{\prime \prime}\right\|_{0}+\left\|\partial_{t} v^{\prime}\right\|_{0}\right] t \sup _{[0, t]}\|\tilde{v}\|_{2} \leq C t P\left(\sup _{[0, t]} E\right) .
\end{aligned}
$$


Similarly, the fifth term of the right-hand side of (6.12) yields the following estimate:

$$
\begin{aligned}
\left\|\rho_{0} \partial_{t}^{2} \tilde{v}^{\prime} \frac{\tilde{\eta}^{\prime \prime}}{\tilde{\eta}^{\prime}}\right\|_{0}(t) \leq C\left\|\rho_{0} \partial_{t}^{2} \tilde{v}^{\prime}\right\|_{L \infty}\left\|\tilde{\eta}^{\prime \prime}\right\|_{0} & \leq C\left\|\rho_{0} \partial_{t}^{2} \tilde{v}^{\prime}\right\|_{1}\left\|\int_{0} \cdot \tilde{v}^{\prime \prime}\right\|_{0} \\
& \leq C t P\left(\sup _{[0, t]} E\right) .
\end{aligned}
$$

Combining estimates (6.14)-(6.17), we obtain the inequality

$$
\sup _{[0, t]}\left\|\frac{2}{\rho_{0}}\left[\rho_{0}^{2} \partial_{t}^{2} \tilde{v}^{\prime}\right]^{\prime}\right\|_{0} \leq C t P\left(\sup _{[0, t]} E\right)+C \sup _{[0, t]} E^{3 / 4}\left(M_{0}+t P\left(\sup _{[0, t]} E\right)\right) .
$$

At this stage, we remind the reader that the solution $\tilde{v}$ to our parabolic $\kappa$-problem is in $X_{T_{K}}$, so that for any $t \in\left[0, T_{\kappa}\right], \partial_{t}^{2} \tilde{v} \in H^{2}(I)$. Notice that

$$
\frac{1}{\rho_{0}}\left[\rho_{0}^{2} \partial_{t}^{2} \tilde{v}^{\prime}\right]^{\prime}=\rho_{0} \partial_{t}^{2} \tilde{v}^{\prime \prime}+2 \rho_{0}^{\prime} \partial_{t}^{2} \tilde{v}^{\prime}
$$

so (6.18) is equivalent to

$$
\begin{aligned}
\sup _{[0, t]}\left\|\rho_{0} \partial_{t}^{2} \tilde{v}^{\prime \prime}+2 \rho_{0}^{\prime} \partial_{t}^{2} \tilde{v}^{\prime} d\right\|_{0} \leq \\
C t P\left(\sup _{[0, t]} E\right)+C \sup _{[0, t]} E^{3 / 4}\left(M_{0}+t P\left(\sup _{[0, t]} E\right)\right) .
\end{aligned}
$$

From this inequality, we would like to conclude that both $\left\|\partial_{t}^{2} \tilde{v}^{\prime}\right\|_{0}$ and $\left\|\rho_{0} \partial_{t}^{2} \tilde{v}^{\prime \prime}\right\|_{0}$ are bounded by the right-hand side of (6.19); the regularity provided by solutions of the $\kappa$-problem allow us to arrive at this conclusion.

By expanding the left-hand side of (6.19), we see that

$$
\begin{aligned}
\sup _{[0, t]}\left\|\rho_{0} \partial_{t}^{2} \tilde{v}^{\prime \prime}+2 \rho_{0}^{\prime} \partial_{t}^{2} \tilde{v}^{\prime}\right\|_{0}^{2}= & \left\|\rho_{0} \partial_{t}^{2} \tilde{v}^{\prime \prime}\right\|_{0}^{2}+4\left\|\rho_{0}^{\prime} \partial_{t}^{2} \tilde{v}^{\prime}\right\|_{0}^{2} \\
& +4 \int_{I} \rho_{0} \partial_{t}^{2} \tilde{v}^{\prime \prime} \rho_{0}^{\prime} \partial_{t}^{2} \tilde{v}^{\prime}
\end{aligned}
$$

Given the regularity of $\partial_{t}^{2} \tilde{v}$ provided by our parabolic $\kappa$-problem, we notice that the cross-term in (6.20) is an exact derivative,

$$
4 \int_{I} \rho_{0} \partial_{t}^{2} \tilde{v}^{\prime \prime} \rho_{0}^{\prime} \partial_{t}^{2} \tilde{v}^{\prime}=2 \int_{I} \rho_{0} \rho_{0}^{\prime} \frac{\partial}{\partial x}\left|\partial_{t}^{2} \tilde{v}^{\prime}\right|^{2},
$$

so that by integrating by parts, we find that

$$
4 \int_{I} \rho_{0} \partial_{t}^{2} \tilde{v}^{\prime \prime} \rho_{0}^{\prime} \partial_{t}^{2} \tilde{v}^{\prime}=-2\left\|\rho_{0}^{\prime} \partial_{t}^{2} \tilde{v}^{\prime}\right\|_{0}^{2}-\int_{I} \rho_{0} \partial_{t}^{2} \tilde{v}^{\prime} \rho_{0}^{\prime \prime} \partial_{t}^{2} \tilde{v}^{\prime}
$$


and hence (6.20) becomes

$$
\begin{aligned}
& \sup _{[0, t]}\left\|\rho_{0} \partial_{t}^{2} \tilde{v}^{\prime \prime}+2 \rho_{0}^{\prime} \partial_{t}^{2} \tilde{v}^{\prime}\right\|_{0}^{2}=\left\|\rho_{0} \partial_{t}^{2} \tilde{v}^{\prime \prime}\right\|_{0}^{2}+2\left\|\rho_{0}^{\prime} \partial_{t}^{2} \tilde{v}^{\prime}\right\|_{0}^{2} \\
& -\int_{I} \rho_{0} \partial_{t}^{2} \tilde{v}^{\prime} \rho_{0}^{\prime \prime} \partial_{t}^{2} \tilde{v}^{\prime}
\end{aligned}
$$

Since the energy function $E$ contains $\rho_{0} \partial_{t}^{2} \tilde{v}(t) \in H^{2}(I)$ and $\partial_{t}^{2} \tilde{v}(t) \in H^{1}(I)$, the fundamental theorem of calculus shows that

$$
\int_{I} \rho_{0} \partial_{t}^{2} \tilde{v}^{\prime} \rho_{0}^{\prime \prime} \partial_{t}^{2} \tilde{v}^{\prime} \leq C t P\left(\sup _{[0, t]} E\right)+C \sup _{[0, t]} E^{3 / 4}\left(M_{0}+t P\left(\sup _{[0, t]} E\right)\right) .
$$

Combining this inequality with (6.21) and (6.18) yields

$$
\begin{aligned}
\sup _{[0, t]}\left[\left\|\rho_{0} \partial_{t}^{2} \tilde{v}^{\prime \prime}\right\|_{0}+\left\|\rho_{0}^{\prime} \partial_{t}^{2} \tilde{v}^{\prime}\right\|_{0}\right] \leq & C t P\left(\sup _{[0, t]} E\right) \\
& +C \sup _{[0, t]} E^{3 / 4}\left(M_{0}+t P\left(\sup _{[0, t]} E\right)\right),
\end{aligned}
$$

and thus

$$
\begin{gathered}
\sup _{[0, t]}\left[\left\|\rho_{0} \partial_{t}^{2} \tilde{v}^{\prime \prime}\right\|_{0}+\left\|\rho_{0}^{\prime} \partial_{t}^{2} \tilde{v}^{\prime}\right\|_{0}+\left\|\rho_{0} \partial_{t}^{2} \tilde{v}^{\prime}\right\|_{0}\right] \leq \\
M_{0}+C t P\left(\sup _{[0, t]} E\right)+C \sup _{[0, t]} E^{3 / 4}\left(M_{0}+t P\left(\sup _{[0, t]} E\right)\right),
\end{gathered}
$$

and hence with the physical vacuum conditions on $\rho_{0}$ given by (1.5) and (1.6), we have that

$$
\begin{aligned}
& \sup _{[0, t]}\left[\left\|\rho_{0} \partial_{t}^{2} \tilde{v}^{\prime \prime}\right\|_{0}+\left\|\partial_{t}^{2} \tilde{v}^{\prime}\right\|_{0}\right] \leq \\
& M_{0}+C t P\left(\sup _{[0, t]} E\right)+C \sup _{[0, t]} E^{3 / 4}\left(M_{0}+t P\left(\sup _{[0, t]} E\right)\right),
\end{aligned}
$$

which, together with (6.13), provides us with the estimate

$$
\begin{aligned}
\sup _{[0, t]}\left[\left\|\rho_{0} \partial_{t}^{2} \tilde{v}^{\prime \prime}\right\|_{0}+\left\|\partial_{t}^{2} \tilde{v}\right\|_{1}\right] \leq \\
M_{0}+C t P\left(\sup _{[0, t]} E\right)+C \sup _{[0, t]} E^{3 / 4}\left(M_{0}+t P\left(\sup _{[0, t]} E\right)\right) .
\end{aligned}
$$

\subsection{Elliptic and Hardy-Type Estimates for $v(t)$}

Having obtained the estimates for $\partial_{t}^{2} \tilde{v}(t)$ in (6.22), we can next obtain our estimates for $\tilde{v}(t)$. To do so, we consider the first time-differentiated version of (4.5a), which yields the equation

$$
-2\left[\frac{\rho_{0}^{2} \tilde{v}^{\prime}}{\tilde{\eta}^{\prime 3}}\right]^{\prime}-\kappa\left[\rho_{0}^{2} \partial_{t} \tilde{v}^{\prime}\right]^{\prime}=-\rho_{0} \partial_{t}^{2} \tilde{v}
$$


which we rewrite as the following identity:

$$
-\frac{2}{\rho_{0}}\left[\rho_{0}^{2} \tilde{v}^{\prime}\right]^{\prime}-\frac{\kappa}{\rho_{0}}\left[\rho_{0}^{2} \partial_{t} \tilde{v}^{\prime}\right]^{\prime}=-\partial_{t}^{2} \tilde{v}-\frac{2}{\rho_{0}}\left[\rho_{0}^{2} \tilde{v}^{\prime}\right]^{\prime}\left(1-\frac{1}{\tilde{\eta}^{\prime 3}}\right) .
$$

Using Lemma 3.2, we see that for any $t \in\left[0, T_{\kappa}\right]$,

$$
\begin{aligned}
\sup _{[0, t]}\left\|\frac{1}{\rho_{0}}\left[\rho_{0}^{2} \tilde{v}^{\prime}\right]^{\prime}\right\|_{1} \leq & C \sup _{[0, t]}\left\|\partial_{t}^{2} \tilde{v}\right\|_{1}+C \sup _{[0, t]}\left\|\frac{1}{\rho_{0}}\left[\rho_{0}^{2} \tilde{v}^{\prime}\right]^{\prime}\right\|_{1}\left\|\int_{0}^{\cdot} \frac{\tilde{v}^{\prime}}{\tilde{\eta}^{\prime 4}}\right\|_{L^{\infty}} \\
& +C\left\|\frac{1}{\rho_{0}}\left[\rho_{0}^{2} \tilde{v}^{\prime}\right]^{\prime} \frac{\tilde{\eta}^{\prime \prime}}{\tilde{\eta}^{\prime 4}}\right\|_{0}
\end{aligned}
$$

We next estimate each term on the right-hand side of (6.24). The bound for the first term on the right-hand side of (6.24) is provided by (6.22). The second term of the right-hand side of (6.24) is estimated as follows:

$$
\begin{aligned}
\left\|\frac{1}{\rho_{0}}\left[\rho_{0}^{2} \tilde{v}^{\prime}\right]^{\prime}\right\|_{1}\left\|\int_{0}^{\cdot} \frac{\tilde{v}^{\prime}}{\tilde{\eta}^{\prime 4}}\right\|_{L^{\infty}}(t) & \leq C\left[\left\|\rho_{0} \tilde{v}^{\prime \prime \prime}\right\|_{0}+\|v\|_{2}\right] t \sup _{[0, t]}\|\tilde{v}\|_{2} \\
& \leq C t P\left(\sup _{[0, t]} E\right) .
\end{aligned}
$$

For the third term on the right-hand side of (6.24),

$$
\begin{aligned}
\left\|\frac{1}{\rho_{0}}\left[\rho_{0}^{2} \tilde{v}^{\prime}\right]^{\prime}\left[\frac{\tilde{\eta}^{\prime \prime}}{\tilde{\eta}^{\prime 4}}\right]\right\|_{1}(t) & \leq C\left\|\frac{1}{\rho_{0}}\left[\rho_{0}^{2} \tilde{v}^{\prime}\right]^{\prime}\right\|_{L^{\infty}}\left\|\tilde{\eta}^{\prime \prime}\right\|_{0} \\
& \leq C\left[\left\|\rho_{0} \tilde{v}^{\prime \prime \prime}\right\|_{0}+\|v\|_{2}\right]\left\|\int_{0}^{t} \tilde{v}^{\prime \prime}\right\| \\
& \leq C t P\left(\sup _{[0, t]} E\right) .
\end{aligned}
$$

Combining these estimates provides the inequality

$$
\sup _{[0, t]}\left\|\frac{1}{\rho_{0}}\left[\rho_{0}^{2} \tilde{v}^{\prime}\right]^{\prime}\right\|_{1} \leq C t P\left(\sup _{[0, t]} E\right)+C \sup _{[0, t]} E^{3 / 4}\left(M_{0}+t P\left(\sup _{[0, t]} E\right)\right),
$$

which leads us immediately to

$$
\sup _{[0, t]}\left\|\rho_{0} \tilde{v}^{\prime \prime \prime}+3 \rho_{0}^{\prime} v^{\prime \prime}\right\|_{0} \leq C t P\left(\sup _{[0, t]} E\right)+C \sup _{[0, t]} E^{3 / 4}\left(M_{0}+t P\left(\sup _{[0, t]} E\right)\right)
$$


Now, since for any $t \in\left[0, T_{\kappa}\right]$, the solution $\tilde{v}$ to our parabolic $\kappa$-problem is in $H^{3}(I)$, we infer that $\rho_{0} v^{\prime \prime \prime} \in L^{2}(I)$. We can then apply the same integration-byparts argument as in [1] to find that

$$
\begin{aligned}
\left\|\rho_{0} \tilde{v}^{\prime \prime \prime}+3 \rho_{0}^{\prime} v^{\prime \prime}\right\|_{0}^{2} & =\left\|\rho_{0} \tilde{v}^{\prime \prime \prime}\right\|_{0}^{2}+9\left\|\rho_{0}^{\prime} \tilde{v}^{\prime \prime}\right\|_{0}^{2}+3 \int_{I} \rho_{0} \rho_{0}^{\prime}\left[\left|\tilde{v}^{\prime \prime}\right|^{2}\right]^{\prime} \\
& =\left\|\rho_{0} \tilde{v}^{\prime \prime \prime}\right\|_{0}^{2}+9\left\|\rho_{0}^{\prime} \tilde{v}^{\prime \prime}\right\|_{0}^{2}-3 \int_{I}\left[\rho_{0} \rho_{0}^{\prime \prime}+\rho_{0}^{\prime 2}\right]\left|\tilde{v}^{\prime \prime}\right|^{2} \\
& =\left\|\rho_{0} \tilde{v}^{\prime \prime \prime}\right\|_{0}^{2}+6\left\|\rho_{0}^{\prime} \tilde{v}^{\prime \prime}\right\|_{0}^{2}-3 \int_{I} \rho_{0} \rho_{0}^{\prime \prime}\left|\tilde{v}^{\prime \prime}\right|^{2}
\end{aligned}
$$

Combined with (6.27), this yields

$$
\begin{aligned}
\sup _{[0, t]}\left[\left\|\rho_{0} \tilde{v}^{\prime \prime \prime}\right\|_{0}+\left\|\rho_{0}^{\prime} \tilde{v}^{\prime \prime}\right\|_{0}\right] \leq & C t P\left(\sup _{[0, t]} E\right)+C \sup _{[0, t]} E^{3 / 4}\left(M_{0}+t P\left(\sup _{[0, t]} E\right)\right) \\
& +M_{0}+C\left\|\int_{0}^{t} \sqrt{\rho_{0}} \tilde{v}_{t}^{\prime \prime}\right\|_{0},
\end{aligned}
$$

and thus

$$
\begin{aligned}
\sup _{[0, t]}\left[\left\|\rho_{0} \tilde{v}^{\prime \prime \prime}\right\|_{0}+\left\|\rho_{0}^{\prime} \tilde{v}^{\prime \prime}\right\|_{0}+\left\|\rho_{0} \tilde{v}^{\prime \prime}\right\|_{0}\right] \leq \\
M_{0}+C t P\left(\sup _{[0, t]} E\right)+C \sup _{[0, t]} E^{3 / 4}\left(M_{0}+t P\left(\sup _{[0, t]} E\right)\right),
\end{aligned}
$$

With (1.5) and (1.6), it follows that

$$
\begin{aligned}
& \sup _{[0, t]}\left[\left\|\rho_{0} \tilde{v}^{\prime \prime \prime}\right\|_{0}+\left\|\tilde{v}^{\prime \prime}\right\|_{0}\right] \leq \\
& M_{0}+C t P\left(\sup _{[0, t]} E\right)+C \sup _{[0, t]} E^{3 / 4}\left(M_{0}+t P\left(\sup _{[0, t]} E\right)\right),
\end{aligned}
$$

and hence

$$
\begin{aligned}
\sup _{[0, t]}\left[\left\|\rho_{0} \tilde{v}^{\prime \prime \prime}\right\|_{0}+\|\tilde{v}\|_{2}\right] \leq & M_{0}+C t P\left(\sup _{[0, t]} E\right) \\
& +C \sup _{[0, t]} E^{3 / 4}\left(M_{0}+t P\left(\sup _{[0, t]} E\right)\right) .
\end{aligned}
$$

\subsection{Elliptic and Hardy-Type Estimates for $\partial_{t}{ }_{t} v(t)$ and $\partial_{t} v(t)$}

We consider the fourth time-differentiated version of (4.5a):

$$
\left[\partial_{t}^{4} \frac{\rho_{0}^{2}}{\tilde{\eta}^{\prime 2}}\right]^{\prime}-\kappa\left[\rho_{0}^{2} \partial_{t}^{4} \tilde{v}^{\prime}\right]^{\prime}=-\rho_{0} \partial_{t}^{5} \tilde{v}
$$


which can be rewritten as

$$
-2\left[\frac{\rho_{0}^{2} \partial_{t}^{3} \tilde{v}^{\prime}}{\tilde{\eta}^{\prime 3}}\right]^{\prime}-\kappa\left[\rho_{0}^{2} \partial_{t}^{4} \tilde{v}^{\prime}\right]^{\prime}=-\rho_{0} \partial_{t}^{5} \tilde{v}+c_{1}\left[\frac{\rho_{0}^{2} \partial_{t}^{2} \tilde{v}^{\prime} \tilde{v}^{\prime}}{\tilde{\eta}^{\prime 4}}\right]^{\prime}+c_{2}\left[\frac{\rho_{0}^{3 / 2} \partial_{t} \tilde{v}^{\prime 2}}{\tilde{\eta}^{\prime 5}}\right]^{\prime}
$$

for some constants $c_{1}$ and $c_{2}$. By employing the fundamental theorem of calculus and dividing by $\rho_{0}^{1 / 2}$, we obtain the equation

$$
\begin{aligned}
& -\frac{2}{\rho_{0}^{1 / 2}}\left[\rho_{0}^{2} \partial_{t}^{3} \tilde{v}^{\prime}\right]^{\prime}-\frac{\kappa}{\rho_{0}^{1 / 2}}\left[\rho_{0}^{2} \partial_{t}^{4} \tilde{v}^{\prime}\right]^{\prime} \\
& =-\sqrt{\rho_{0}} \partial_{t}^{5} \tilde{v}+\frac{c_{1}}{\rho_{0}^{1 / 2}}\left[\frac{\rho_{0}^{2} \partial_{t}^{2} \tilde{v}^{\prime} \tilde{v}^{\prime}}{\tilde{\eta}^{\prime 4}}\right]^{\prime}+\frac{c_{2}}{\rho_{0}^{1 / 2}}\left[\frac{\rho_{0}^{2} \partial_{t} \tilde{v}^{\prime 2}}{\tilde{\eta}^{\prime 5}}\right]^{\prime} \\
& \quad-\frac{2}{\rho_{0}^{1 / 2}}\left[\rho_{0}^{2} \partial_{t}^{3} \tilde{v}^{\prime}\right]^{\prime}\left(1-\frac{1}{\tilde{\eta}^{\prime 3}}\right)-6 \rho_{0}^{3 / 2} \partial_{t}^{3} \tilde{v}^{\prime} \frac{\tilde{\eta}^{\prime \prime}}{\tilde{\eta}^{\prime 4}} .
\end{aligned}
$$

For any $t \in\left[0, T_{\kappa}\right]$, Lemma 3.2 provides the $\kappa$-independent estimate

$$
\begin{aligned}
\sup _{[0, t]}\left\|\frac{2}{\rho_{0}^{1 / 2}}\left[\rho_{0}^{2} \partial_{t}^{3} \tilde{v}^{\prime}\right]^{\prime}\right\|_{0} \\
\leq \sup _{[0, t]}\left\|\sqrt{\rho_{0}} \partial_{t}^{5} \tilde{v}\right\|_{0}+\sup _{[0, t]}\left\|\frac{c_{1}}{\rho_{0}^{1 / 2}}\left[\frac{\rho_{0}^{2} \partial_{t}^{2} \tilde{v}^{\prime} \tilde{v}^{\prime}}{\tilde{\eta}^{\prime 4}}\right]^{\prime}\right\|_{0} \\
\quad+\sup _{[0, t]}\left\|\frac{c_{2}}{\rho_{0}^{1 / 2}}\left[\frac{\rho_{0}^{2} \partial_{t} \tilde{v}^{\prime 2}}{\tilde{\eta}^{\prime 5}}\right]^{\prime}\right\|_{0} \\
\quad+\sup _{[0, t]}\left\|\frac{2}{\rho_{0}^{1 / 2}}\left[\rho_{0}^{2} \partial_{t}^{3} \tilde{v}^{\prime}\right]^{\prime}\right\|_{0}\left\|3 \int_{0} \frac{\tilde{v}^{\prime}}{\tilde{\eta}^{\prime 4}}\right\|_{L^{\infty}}+6 \sup _{[0, t]}\left\|\rho_{0}^{3 / 2} \partial_{t}^{2} \tilde{v}^{\prime} \frac{\tilde{\eta}^{\prime \prime}}{\tilde{\eta}^{\prime 4}}\right\|_{0} .
\end{aligned}
$$

We estimate each term on the right-hand side of (6.30). The first term on the right-hand side is bounded by $M_{0}+C t P\left(\sup _{[0, t]} E\right)$ thanks to (6.10). For the second term on the right-hand side of (6.30) we have that

$$
\begin{aligned}
& \left\|\frac{1}{\rho_{0}^{1 / 2}}\left[\frac{\rho_{0}^{2} \partial_{t}^{2} \tilde{v}^{\prime} \tilde{v}^{\prime}}{\tilde{\eta}^{\prime 4}}\right]^{\prime}\right\|_{0} \\
& \leq\left\|\sqrt{\rho_{0}}\left(\rho_{0} \partial_{t}^{2} \tilde{v}^{\prime}\right)^{\prime}\right\|_{0}\left\|\frac{\tilde{v}^{\prime}}{\tilde{\eta}^{\prime 4}}\right\|_{L^{\infty}}+\left\|\sqrt{\rho_{0}} \partial_{t}^{2} \tilde{v}^{\prime}\left[\frac{\rho_{0} \tilde{v}^{\prime}}{\tilde{\eta}^{\prime 4}}\right]^{\prime}\right\|_{0} \\
& \leq C\left\|\sqrt{\rho_{0}}\left(\rho_{0} \partial_{t}^{2} \tilde{v}^{\prime}\right)^{\prime}\right\|_{0}\left\|\tilde{v}^{\prime}\right\|_{3 / 4}+\left\|\sqrt{\rho_{0}} \partial_{t}^{2} \tilde{v}^{\prime}\left[\frac{\rho_{0}^{\prime} \tilde{v}^{\prime}}{\tilde{\eta}^{\prime 4}}\right]\right\|_{0} \\
& \quad+\left\|\sqrt{\rho_{0}} \partial_{t}^{2} \tilde{v}^{\prime}\left[\frac{\rho_{0} \tilde{v}^{\prime \prime}}{\tilde{\eta}^{\prime 4}}\right]\right\|_{0}+4\left\|\sqrt{\rho_{0}} \partial_{t}^{2} \tilde{v}^{\prime}\left[\frac{\rho_{0} \tilde{v}^{\prime} \tilde{\eta}^{\prime \prime}}{\tilde{\eta}^{\prime 5}}\right]\right\|_{0} \leq
\end{aligned}
$$




$$
\begin{aligned}
& \leq C\left\|\sqrt{\rho_{0}}\left(\rho_{0} \tilde{v}_{2}^{\prime}\right)^{\prime}+\int_{0}^{.} \sqrt{\rho_{0}}\left(\rho_{0} \partial_{t}^{3} \tilde{v}^{\prime}\right)^{\prime}\right\|_{0}\left\|\tilde{v}^{\prime}\right\|_{1}^{1 / 2}\left\|\tilde{v}_{1}^{\prime}+\int_{0}^{\cdot} \tilde{v}_{t}^{\prime}\right\|_{1 / 2}^{1 / 2} \\
& +C\left\|\sqrt{\rho_{0}} \tilde{v}_{2}^{\prime}+\int_{0}^{\cdot} \sqrt{\rho_{0}} \partial_{t}^{3} \tilde{v}^{\prime}\right\|_{0}\left\|\tilde{v}^{\prime}\right\|_{3 / 4}+C\left\|\sqrt{\rho_{0}} \partial_{t}^{2} \tilde{v}^{\prime}\right\|_{0}\left\|\rho_{0} v^{\prime \prime}\right\|_{3 / 4} \\
& +C\left\|\tilde{v}^{\prime}\right\|_{3 / 4}\left\|\int_{0}^{\cdot} \tilde{v}^{\prime \prime}\right\|_{0}\left\|\rho_{0}^{3 / 2} \tilde{v}_{2}^{\prime}+\int_{0}^{\cdot} \rho_{0}^{3 / 2} \partial_{t}^{3} \tilde{v}^{\prime}\right\|_{1} \\
& \leq\left\|\sqrt{\rho_{0}}\left(\rho_{0} \tilde{v}_{2}^{\prime}\right)^{\prime}+\int_{0}^{\cdot} \sqrt{\rho_{0}}\left(\rho_{0} \tilde{v}_{t t}^{\prime}\right)^{\prime}\right\|_{0}\left\|\tilde{v}^{\prime}\right\|_{1}^{1 / 2}\left\|\tilde{v}_{1}^{\prime}+\int_{0}^{\cdot} \tilde{v}_{t}^{\prime}\right\|_{1 / 2}^{1 / 2} \\
& +C\left\|\sqrt{\rho_{0}} \tilde{v}_{2}^{\prime}+\int_{0}^{\cdot} \sqrt{\rho_{0}} \tilde{v}_{t t}^{\prime}\right\|_{0}\left\|\tilde{v}^{\prime}\right\|_{1}^{1 / 2}\left\|\tilde{v}_{1}^{\prime}+\int_{0}^{\cdot} \tilde{v}_{t}^{\prime}\right\|_{1 / 2}^{1 / 2} \\
& +C\left\|\sqrt{\rho_{0}} \partial_{t}^{2} \tilde{v}^{\prime}\right\|_{0}\left\|\rho_{0} v^{\prime \prime}\right\|_{1}^{3 / 4}\left\|\rho_{0} v_{0}^{\prime \prime}+\int_{0}^{\cdot} \rho_{0} v_{t}^{\prime \prime}\right\|_{0}^{1 / 4} \\
& +C\left\|\tilde{v}^{\prime}\right\|_{1}^{1 / 2}\left\|\tilde{v}_{1}^{\prime}+\int_{0}^{\cdot} \tilde{v}_{t}^{\prime}\right\|_{1 / 2}^{1 / 2}\left\|\int_{0}^{\cdot} \tilde{v}^{\prime \prime}\right\|_{0}\left\|\rho_{0}^{3 / 2} \tilde{v}_{2}^{\prime}+\int_{0}^{\cdot} \rho_{0}^{3 / 2} \partial_{t}^{3} \tilde{v}^{\prime}\right\|_{1},
\end{aligned}
$$

where we have again used the fact that $\|\cdot\|_{L^{\infty}} \leq C\|\cdot\|_{3 / 4}$. Thanks to the definition of $E$, the previous inequality shows that for any $t \in\left[0, T_{\kappa}\right]$,

$$
\sup _{[0, t]}\left\|\frac{1}{\sqrt{\rho_{0}}}\left[\frac{\rho_{0}^{2} \partial_{t}^{2} \tilde{v}^{\prime} \tilde{v}^{\prime}}{\tilde{\eta}^{\prime 4}}\right]^{\prime}\right\|_{0} \leq C \sup _{[0, t]} E^{3 / 4}\left(M_{0}+t P\left(\sup _{[0, t]} E\right)\right) .
$$

For the third term on the right-hand side of (6.30), we similarly have that

$$
\begin{aligned}
\| \frac{1}{\sqrt{\rho_{0}}} & {\left[\frac{\rho_{0}^{2} \partial_{t} \tilde{v}^{\prime 2}}{\tilde{\eta}^{\prime 5}}\right]^{\prime} \|_{0}{ }^{(t)} } \\
\leq & 2\left\|\left(\rho_{0} \partial_{t} \tilde{v}^{\prime}\right)^{\prime}\right\|_{0}\left\|\sqrt{\rho_{0}} \frac{\partial_{t} \tilde{v}^{\prime}}{\tilde{\eta}^{\prime 5}}\right\|_{L^{\infty}}+5\left\|\sqrt{\rho_{0}} \frac{\partial_{t} \tilde{v}^{\prime 2}}{\tilde{\eta}^{\prime 6}}\right\|_{L^{1}}\left\|\rho_{0} \tilde{\eta}^{\prime \prime}\right\|_{L^{\infty}} \\
\leq & C\left\|\left(\rho_{0} \tilde{v}_{1}^{\prime}\right)^{\prime}+\int_{0}^{t}\left(\rho_{0} \partial_{t t} \tilde{v}^{\prime}\right)^{\prime}\right\|_{0}\left\|\sqrt{\rho_{0}} \partial_{t} \tilde{v}^{\prime}\right\|_{3 / 4}+C\left\|\partial_{t} \tilde{v}^{\prime}\right\|_{0}^{2}\left\|\int_{0}^{t}\left(\rho_{0} \tilde{v}^{\prime \prime}\right)^{\prime}\right\|_{0} \\
\leq & C\left\|\left(\rho_{0} \tilde{v}_{1}^{\prime}\right)^{\prime}+\int_{0}^{t}\left(\rho_{0} \partial_{t t} \tilde{v}^{\prime}\right)^{\prime}\right\|_{0}\left\|\sqrt{\rho_{0}} \partial_{t} \tilde{v}^{\prime}\right\|_{0}^{1-\alpha}\left\|\left(\sqrt{\rho_{0}} \partial_{t} \tilde{v}^{\prime}\right)^{\prime}\right\|_{L^{2-a}}^{\alpha} \\
& +C\left\|\partial_{t} \tilde{v}^{\prime}\right\|_{0}^{2}\left\|\int_{0}^{t}\left(\rho_{0} \tilde{v}^{\prime \prime}\right)^{\prime}\right\|_{0} \\
\leq & C\left\|\left(\rho_{0} \tilde{v}_{1}^{\prime}\right)^{\prime}+\int_{0}^{t}\left(\rho_{0} \partial_{t t} \tilde{v}^{\prime}\right)^{\prime}\right\|_{0}\left\|\tilde{v}_{1}^{\prime}+\int_{0}^{t} \partial_{t t} \tilde{v}^{\prime}\right\|_{0}^{1-\alpha}\left\|\left(\sqrt{\rho_{0}} \partial_{t} \tilde{v}^{\prime}\right)^{\prime}\right\|_{L^{2-a}}^{\alpha} \\
& +C\left\|\partial_{t} \tilde{v}^{\prime}\right\|_{0}^{2}\left\|\int_{0}^{t}\left(\rho_{0} \tilde{v}^{\prime \prime}\right)^{\prime}\right\|_{0},
\end{aligned}
$$

where $0<a<\frac{1}{2}$ is given and $0<\alpha=\frac{3-3 a}{4+3 a}<1$. 
The only term on the right-hand side of (6.32) that is not directly contained in the definition of $E$ is $\left\|\left(\sqrt{\rho_{0}} \partial_{t} \tilde{v}^{\prime}\right)^{\prime}\right\|_{L^{2-a}}^{\alpha}$. To this end, we notice that

$$
\begin{aligned}
\left\|\left(\sqrt{\rho_{0}} \partial_{t} \tilde{v}^{\prime}\right)^{\prime}\right\|_{L^{2-a}} & \leq\left\|\frac{\partial_{t} \tilde{v}^{\prime}}{2 \sqrt{\rho_{0}}}\right\|_{L^{2-a}}+\left\|\sqrt{\rho_{0}} \tilde{v}_{t t}^{\prime \prime}\right\|_{0} \\
& \leq\left\|\frac{1}{2 \sqrt{\rho_{0}}}\right\|_{L^{2-\frac{a}{2}}}\left\|\partial_{t} \tilde{v}^{\prime}\right\|_{1 / 2}+\left\|\sqrt{\rho_{0}} \tilde{v}_{t t}^{\prime \prime}\right\|_{0},
\end{aligned}
$$

where we have used the fact that $\|\cdot\|_{L^{p}} \leq C_{p}\|\cdot\|_{1 / 2}$ for all $1<p<\infty$. Thanks to the definition of $E$, the previous inequality and (6.32) provides us with, for any $t \in\left[0, T_{\kappa}\right]$,

$$
\sup _{[0, t]}\left\|\frac{1}{\rho_{0}}\left[\frac{\rho_{0}^{2} \tilde{v}^{\prime 3}}{\tilde{\eta}^{\prime 5}}\right]^{\prime}\right\|_{0} \leq C \sup _{[0, t]} E^{\alpha}\left(M_{0}+t P\left(\sup _{[0, t]} E\right)\right),
$$

where we again recall that $0<\alpha=\frac{3-3 a}{4+3 a}<1$.

The fourth term on the right-hand side of (6.30) is easily treated:

$$
\begin{aligned}
& \left\|\frac{1}{\sqrt{\rho_{0}}}\left[\rho_{0}^{2} \partial_{t}^{3} \tilde{v}^{\prime}\right]^{\prime}\right\|_{0}\left\|\int_{0}^{t} \frac{\tilde{v}^{\prime}}{\tilde{\eta}^{\prime 4}}\right\|_{L^{\infty}}(t) \\
& \leq C\left[\left\|\rho_{0}^{3 / 2} \partial_{t}^{3} v^{\prime \prime}\right\|_{0}+\left\|\sqrt{\rho_{0}} \partial_{t}^{3} v^{\prime}\right\|_{0}\right] t \sup _{[0, t]}\|\tilde{v}\|_{2} \\
& \quad \leq \operatorname{CtP}\left(\sup _{[0, t]} E\right) .
\end{aligned}
$$

Similarly, the fifth term on the right-hand side of (6.30) is estimated as follows:

$$
\begin{aligned}
\left\|\rho_{0}^{3 / 2} \partial_{t}^{3} \tilde{v}^{\prime} \frac{\tilde{\eta}^{\prime \prime}}{\tilde{\eta}^{\prime 4}}\right\|_{0}(t) & \leq C\left\|\rho_{0}^{3 / 2} \partial_{t}^{3} \tilde{v}^{\prime}\right\|_{L^{\infty}}\left\|\tilde{\eta}^{\prime \prime}\right\|_{0} \\
& \leq C\left[\left\|\rho_{0}^{3 / 2} \partial_{t}^{3} v^{\prime \prime}\right\|_{0}+\left\|\sqrt{\rho_{0}} \partial_{t}^{3} v^{\prime}\right\|_{0}\right]\left\|\int_{0}^{t} \tilde{v}^{\prime \prime}\right\|_{0} \\
& \leq C t P\left(\sup _{[0, t]} E\right) .
\end{aligned}
$$

Combining estimates (6.30)-(6.36), we can infer that

$$
\sup _{[0, t]}\left\|\frac{1}{\rho_{0}^{1 / 2}}\left[\rho_{0}^{2} \partial_{t}^{3} \tilde{v}^{\prime}\right]^{\prime}\right\|_{0} \leq C t P\left(\sup _{[0, t]} E\right)+C \sup _{[0, t]} E^{\alpha}\left(M_{0}+t P\left(\sup _{[0, t]} E\right)\right) \text {. }
$$


Now, since for any $t \in\left[0, T_{\kappa}\right]$, solutions to our parabolic $\kappa$-problem have the regularity $\partial_{t}^{2} v \in H^{2}(I)$, we integrate by parts:

$$
\begin{aligned}
& \left\|\frac{1}{\rho_{0}^{1 / 2}}\left[\rho_{0}^{2} \partial_{t}^{3} \tilde{v}^{\prime}\right]^{\prime}\right\|_{0}^{2} \\
& \quad=\left\|\rho_{0}^{3 / 2} \partial_{t}^{3} \tilde{v}^{\prime \prime}\right\|_{0}^{2}+4\left\|\rho_{0}^{1 / 2} \rho_{0}^{\prime} \partial_{t}^{3} \tilde{v}^{\prime}\right\|_{0}^{2}+2 \int_{I} \rho_{0}^{\prime} \rho_{0}^{2}\left[\left|\partial_{t}^{3} \tilde{v}^{\prime}\right|^{2}\right]^{\prime} \\
& \quad=\left\|\rho_{0}^{3 / 2} \partial_{t}^{3} \tilde{v}^{\prime \prime}\right\|_{0}^{2}+4\left\|\rho_{0}^{\prime} \rho_{0}^{1 / 2} \partial_{t}^{3} \tilde{v}^{\prime}\right\|_{0}^{2}-4 \int_{I} \rho_{0}^{\prime 2} \rho_{0}\left|\partial_{t}^{3} \tilde{v}^{\prime}\right|^{2}-2 \int_{I} \rho_{0}^{\prime \prime} \rho_{0}^{2}\left|\partial_{t}^{3} \tilde{v}^{\prime}\right|^{2} \\
& \quad=\left\|\rho_{0}^{3 / 2} \partial_{t}^{3} \tilde{v}^{\prime \prime}\right\|_{0}^{2}-2 \int_{I} \rho_{0}^{\prime \prime} \rho_{0}^{2}\left|\partial_{t}^{3} \tilde{v}^{\prime}\right|^{2} .
\end{aligned}
$$

Combining this with (6.37) and using the fact that $\rho_{0} \partial_{t}^{3} \tilde{v}^{\prime}=\rho_{0} \tilde{v}_{3}+\int_{0}^{t} \rho_{0} \partial_{t}^{4} \tilde{v}^{\prime}$ for the second term on the right-hand side of (6.38), we find that

$$
\sup _{[0, t]}\left\|\rho_{0}^{3 / 2} \partial_{t}^{3} \tilde{v}^{\prime \prime}\right\|_{0} \leq C t P\left(\sup _{[0, t]} E\right)+C \sup _{[0, t]} E^{\alpha}\left(M_{0}+t P\left(\sup _{[0, t]} E\right)\right) .
$$

Now, since

$$
\frac{1}{\rho_{0}^{1 / 2}}\left[\rho_{0}^{2} \partial_{t}^{3} \tilde{v}^{\prime}\right]^{\prime}=\rho_{0}^{3 / 2} \partial_{t}^{3} \tilde{v}^{\prime \prime}+2 \rho_{0}{ }^{1 / 2} \rho_{0}^{\prime} \partial_{t}^{3} \tilde{v}^{\prime},
$$

estimates (6.37) and (6.39) also imply that

$$
\sup _{[0, t]}\left\|\rho_{0}{ }^{1 / 2} \rho_{0}^{\prime} \partial_{t}^{3} \tilde{v}^{\prime}\right\|_{0} \leq C t P\left(\sup _{[0, t]} E\right)+C \sup _{[0, t]} E^{\alpha}\left(M_{0}+t P\left(\sup _{[0, t]} E\right)\right) .
$$

Therefore,

$$
\begin{gathered}
\sup _{[0, t]}\left[\left\|\rho_{0}^{3 / 2} \partial_{t}^{3} \tilde{v}^{\prime \prime}\right\|_{0}+\left\|\rho_{0}^{\prime} \rho_{0}^{1 / 2} \partial_{t}^{3} \tilde{v}^{\prime}\right\|_{0}+\left\|\rho_{0}^{3 / 2} \partial_{t}^{3} \tilde{v}^{\prime}\right\|_{0}\right] \leq \\
M_{0}+C t P\left(\sup _{[0, t]} E\right)+C \sup _{[0, t]} E^{\alpha}\left(M_{0}+t P\left(\sup _{[0, t]} E\right)\right),
\end{gathered}
$$

so that with (1.5) and (1.6),

$$
\begin{gathered}
\sup _{[0, t]}\left[\left\|\rho_{0}^{3 / 2} \partial_{t}^{3} \tilde{v}^{\prime \prime}\right\|_{0}+\left\|\rho_{0}^{1 / 2} \partial_{t}^{3} \tilde{v}^{\prime}\right\|_{0}\right] \leq \\
M_{0}+C t P\left(\sup _{[0, t]} E\right)+C \sup _{[0, t]} E^{\alpha}\left(M_{0}+t P\left(\sup _{[0, t]} E\right)\right) .
\end{gathered}
$$

Together with (6.13) and the weighted embedding estimate (2.1), the above inequality shows that

$$
\begin{gathered}
\sup _{[0, t]}\left[\left\|\rho_{0}^{3 / 2} \partial_{t}^{3} \tilde{v}^{\prime \prime}\right\|_{0}+\left\|\partial_{t}^{3} \tilde{v}\right\|_{1 / 2}\right] \leq \\
M_{0}+C t P\left(\sup _{[0, t]} E\right)+C \sup _{[0, t]} E^{\alpha}\left(M_{0}+t P\left(\sup _{[0, t]} E\right)\right) .
\end{gathered}
$$


By studying the second time-differentiated version of (4.5a) in the same manner, we find that

$$
\begin{aligned}
\sup _{[0, t]}\left[\left\|\rho_{0}^{3 / 2} \partial_{t} \tilde{v}^{\prime \prime \prime}\right\|_{0}+\left\|\partial_{t} \tilde{v}\right\|_{3 / 2}\right] \leq \\
M_{0}+C t P\left(\sup _{[0, t]} E\right)+C \sup _{[0, t]} E^{\alpha}\left(M_{0}+t P\left(\sup _{[0, t]} E\right)\right) .
\end{aligned}
$$

\section{Proof of Theorem 1.1}

\subsection{Time of Existence and Bounds Independent of $\kappa$ and Existence of Solutions to (1.11)}

Summing inequalities (6.10), (6.22), (6.29), (6.41), and (6.42), we find that

$$
\sup _{t \in[0, T]} E(t) \leq M_{0}+C t P\left(\sup _{[0, t]} E\right)+C \sup _{[0, t]} E^{\alpha}\left(M_{0}+t P\left(\sup _{[0, t]} E\right)\right) .
$$

As $\alpha<1$, by employing Young's inequality and readjusting the constants, we obtain

$$
\sup _{t \in[0, T]} E(t) \leq M_{0}+C T P\left(\sup _{t \in[0, T]} E(t)\right) .
$$

Just as in [2, sec. 9], this provides us with a time of existence $T_{1}$ independent of $\kappa$ and an estimate on $\left(0, T_{1}\right)$ independent of $\kappa$ of the type

$$
\sup _{t \in\left[0, T_{1}\right]} E(t) \leq 2 M_{0}
$$

In particular, our sequence of solutions $\left(v_{\kappa}\right)$ satisfies the $\kappa$-independent bound (7.1) on the $\kappa$-independent time interval $\left(0, T_{1}\right)$.

\subsection{Limit as $\kappa \rightarrow 0$}

By the $\kappa$-independent estimate (7.1), there exists a subsequence of $\left\{v_{\kappa}\right\}$ that converges weakly to $v$ in $L^{2}\left(0, T ; H^{2}(I)\right)$. With $\eta(t, x)=x+\int_{0}^{t} v(s, x) d s$, by standard compactness arguments we see that a further subsequence of $v_{\kappa}$ and $\eta_{\kappa}^{\prime}$ uniformly converges to $v$ and $\eta^{\prime}$, respectively, which shows that $v$ is the solution to (1.11) and $v(0, x)=u_{0}(x)$.

\subsection{Uniqueness of Solutions to Compressible Euler Equations (1.11)}

For uniqueness, we require the initial data to have one space derivative better regularity than for existence. Given assumption (1.15) on the data $\left(u_{0}, \rho_{0}\right)$, repeating our argument for existence, we can produce a solution $v$ on $\left[0, T_{1}\right]$ that satisfies the estimate

$$
\sum_{s=0}^{3}\left[\left\|\partial_{t}^{2 s} v(t, \cdot)\right\|_{H^{3-s}(I)}^{2}+\left\|\rho_{0} \partial_{t}^{2 s} v(t, \cdot)\right\|_{H^{4-s}(I)}^{2}\right]<\infty
$$


and has the flow $\eta(t, x)=x+\int_{0}^{t} v(s, x) d s$. For the sake of contradiction, let us assume that $w$ is also a solution on $\left[0, T_{1}\right]$ with initial data $\left(u_{0}, \rho_{0}\right)$, satisfying the same estimate, with flow $\psi(t, x)=x+\int_{0}^{t} w(s, x) d s$.

We define

$$
\delta v=v-w,
$$

in which case we have the following equation for $\delta v$ :

$$
\begin{aligned}
& \rho_{0} \delta v_{t}+\left(\rho_{0}^{2}\left[\eta^{\prime-2}-\psi^{\prime-2}\right]\right)^{\prime}=0 \quad \text { in } I \times\left(0, T_{1}\right], \\
& \delta v=0 \quad \text { on } I \times\{t=0\}, \\
& \rho_{0}=0 \quad \text { on } \partial I \text {. }
\end{aligned}
$$

By considering the fifth time-differentiated version of (7.2a) and taking the $L^{2}(I)$ inner product with $\partial_{t}^{5} \delta v$, we obtain the analogue of (6.10) (with $\kappa=0$ ) for $\delta v$. The additional error terms that arise are easily controlled by the fact that both $v$ and $w$ have one space derivative better regularity than the energy function $E$. This produces a good bound for $\partial_{t}^{4} \delta v \in L^{\infty}\left(0, T_{1} ; L^{2}(I)\right)$. By repeating the elliptic and Hardy-type estimates for $\partial_{t}^{2} \delta v \in L^{\infty}\left(0, T_{1} ; H^{1}(I)\right)$ and $\delta v \in L^{\infty}\left(0, T_{1} ; H^{2}(I)\right)$ and using (7.2b), we obtain the inequality

$$
\begin{aligned}
& \sup _{t \in\left[0, T_{1}\right]}\left(\left\|\partial_{t}^{4} \delta v(t)\right\|_{0}^{2}+\left\|\partial_{t}^{2} \delta v(t)\right\|_{1}^{2}+\|\delta v(t)\|_{2}^{2}\right) \leq \\
& C T_{1} P\left(\sup _{t \in\left[0, T_{1}\right]}\left(\left\|\partial_{t}^{4} \delta v(t)\right\|_{0}^{2}+\left\|\partial_{t}^{2} \delta v(t)\right\|_{1}^{2}+\|\delta v(t)\|_{2}^{2}\right)\right),
\end{aligned}
$$

which shows that $\delta v=0$.

\subsection{Optimal Regularity for Initial Data}

We smoothed our initial data $\left(u_{0}, \rho_{0}\right)$ in order to construct solutions to our degenerate parabolic $\kappa$-problem (4.5). Having obtained solutions that depend only on $E(0, v)$, a standard density argument shows that the initial data only needs to satisfy $M_{0}<\infty$.

\section{The Case $\gamma \neq 2$}

In this section, we describe the modifications to the energy function and the methodology for the case that $\gamma \neq 2$. We denote by $a_{0}$ the integer satisfying the inequality

$$
1<1+\frac{1}{\gamma-1}-a_{0} \leq 2
$$

Letting

$$
d(x)=\operatorname{dist}(x, \partial I)
$$


we consider the following higher-order energy function:

$$
\begin{aligned}
E_{\gamma}(t, v)= & \sum_{s=0}^{4}\left\|\partial_{t}^{s} v(t, \cdot)\right\|_{2-\frac{s}{2}}^{2}+\sum_{s=0}^{2}\left\|d \partial_{t}^{2 s} v(t, \cdot)\right\|_{3-s}^{2} \\
& +\left\|\sqrt{d} \partial_{t} v^{\prime \prime}(t, \cdot)\right\|_{0}^{2}+\left\|\sqrt{d} \partial_{t}^{3} v^{\prime}(t, \cdot)\right\|_{0}^{2} \\
& +\sum_{a=0}^{a_{0}}\left\|\sqrt{d}^{1+\frac{1}{\gamma-1}-a} \partial_{t}^{4+a_{0}-a} v^{\prime}(t, \cdot)\right\|_{0}^{2}
\end{aligned}
$$

and define the polynomial function $M_{0}^{\gamma}=P\left(E_{\gamma}(0, v)\right)$. Notice the last sum in $E_{\gamma}$ appears whenever $\gamma<2$, and the number of time-differentiated problems increases as $\gamma \rightarrow 1$.

We explain this last summation of norms in $E_{\gamma}$ with a particular example; namely, consider the case $\gamma=\frac{3}{2}$. Then $\rho_{0} \sim d^{2}$ near $\Gamma, a_{0}=1$, and the last summation is written as

$$
\sum_{a=0}^{a_{0}}\left\|\sqrt{d}^{1+\frac{1}{\gamma-1}-a} \partial_{t}^{4+a_{0}-a} v^{\prime}(t, \cdot)\right\|_{0}^{2}=\left\|d^{3 / 2} \partial_{t}^{5} v^{\prime}(t)\right\|_{0}^{2}+\left\|d^{1 / 2} \partial_{t}^{4} v^{\prime}(t)\right\|_{0}^{2},
$$

which is equivalent to

$$
\int_{I} \rho_{0}^{3 / 2}\left|\partial_{t}^{5} v^{\prime}(t)\right|^{2} d x+\int_{I} \rho_{0}^{1 / 2}\left|\partial_{t}^{4} v^{\prime}(t)\right|^{2} d x .
$$

The Euler equations with $\gamma=\frac{3}{2}$ are written as

$$
\rho_{0} v_{t}+\left(\rho_{0}^{3 / 2} \eta_{x}^{-3 / 2}\right)_{x}=0
$$

Energy estimates on the sixth time-differentiated problem produce the first integral in (8.1), while the second integral is obtained using our elliptic-type estimates on the fourth time-differentiated version of (8.2). (Notice that the value of $\gamma$ does not play a role in our elliptic-type estimates.) Having control on the two integrals in (8.1) then shows that we are back in the situation for the case that $\gamma \geq 2$; namely, we see that $\partial_{t}^{4} v(t)$ is even better than $L^{2}(I)$, which allows us to proceed as before. In particular, for $\gamma<2$ the power on $\rho_{0}$ in the first integral in (8.1) is greater than 1 , and by weighted embedding estimates, this means that the embedding occurs into a less regular Sobolev space; this accounts for the need to have more time-differentiated problems when $\gamma<2$.

Using the same procedure as we have detailed for the case that $\gamma=2$, we have the following:

THEOREM 8.1 (Existence and Uniqueness for Any $\gamma>1$ ) Given initial data $\left(u_{0}, \rho_{0}\right)$ such that $M_{0}^{\gamma}<\infty, \rho_{0}(x)>0$ for $x \in I$, and the physical vacuum condition (1.4) holds, there exists a solution to (1.10) (and hence (1.1)) on $\left[0, T_{\gamma}\right]$ 
for $T_{\gamma}>0$ taken sufficiently small such that

$$
\sup _{t \in[0, T]} E_{\gamma}(t) \leq 2 M_{0}^{\gamma}
$$

Moreover, if the initial data satisfies

$$
\begin{aligned}
\sum_{s=0}^{3}\left\|\partial_{t}^{s} v(0, \cdot)\right\|_{H^{3-s}(I)}^{2}+\sum_{s=0}^{3}\left\|d \partial_{t}^{2 s} v(0, \cdot)\right\|_{H^{4-s}(I)}^{2} & \\
& +\sum_{a=0}^{a_{0}}\left\|\sqrt{d}^{1+\frac{1}{\gamma-1}-a} \partial_{t}^{6+a_{0}-a} v^{\prime}(0, \cdot)\right\|_{0}^{2}<\infty,
\end{aligned}
$$

then the solution is unique.

Acknowledgment. We are grateful to the anonymous referee for carefully reading our manuscript and making a number of useful suggestions. SS was supported by National Science Foundation Grant DMS-0701056.

\section{Bibliography}

[1] Coutand, D.; Lindblad, H.; Shkoller, S. A priori estimates for the free-boundary 3D compressible Euler equations in physical vacuum. Comm. Math. Phys. 296 (2010), no. 2, 559-587.

[2] Coutand, D.; Shkoller, S. The interaction between quasilinear elastodynamics and the NavierStokes equations. Arch. Ration. Mech. Anal. 179 (2006), no. 3, 303-352.

[3] Coutand, D.; Shkoller, S. Well-posedness of the free-surface incompressible Euler equations with or without surface tension. J. Amer. Math. Soc. 20 (2007), no. 3, 829-930.

[4] Coutand, D.; Shkoller, S. Well-posedness in smooth function spaces for the moving-boundary 3-D compressible Euler equations in physical vacuum. Preprint, 2010. Available at: http:// arxiv.org/abs/1003.4721v3.pdf

[5] Deimling, K. Nonlinear functional analysis. Springer, Berlin, 1985.

[6] Jang, J.; Masmoudi, N. Well-posedness for compressible Euler equations with physical vacuum singularity. Comm. Pure Appl. Math. 62 (2009), no. 10, 1327-1385.

[7] Kufner, A. Weighted Sobolev spaces. Wiley-Interscience, New York, 1985.

[8] Lin, L. W. On the vacuum state for the equations of isentropic gas dynamics. J. Math. Anal. Appl. 121 (1987), no. 2, 406-425.

[9] Lindblad, H. Well posedness for the motion of a compressible liquid with free surface boundary. Comm. Math. Phys. 260 (2005), no. 2, 319-392.

[10] Liu, T.-P. Compressible flow with damping and vacuum. Japan J. Indust. Appl. Math. 13 (1996), no. $1,25-32$.

[11] Liu, T.-P.; Smoller, J. A. On the vacuum state for the isentropic gas dynamics equations. $A d v$. in Appl. Math. 1 (1980), no. 4, 345-359.

[12] Liu, T.-P.; Yang, T. Compressible Euler equations with vacuum. J. Differential Equations 140 (1997), no. 2, 223-237.

[13] Liu, T.-P.; Yang, T. Compressible flow with vacuum and physical singularity. Methods Appl. Anal. 7 (2000), no. 3, 495-510.

[14] Makino, T. On a local existence theorem for the evolution equation of gaseous stars. Patterns and waves, 459-479. Studies in Mathematics and Its Applications, 18. North-Holland, Amsterdam, 1986. 
[15] Masmoudi, N. Video of Discussion: Free boundary problems related to water waves. Summer Program: Nonlinear Conservation Laws and Applications, July 13-31, 2009, Institute for Mathematics and Its Applications, University of Minnesota. Available at: http://www . ima . umn . edu/videos/?id=915

[16] Trakhinin, Y. Local existence for the free boundary problem for nonrelativistic and relativistic compressible Euler equations with a vacuum boundary condition. Comm. Pure Appl. Math. 62 (2009), no. 11, 1551-1594.

[17] Xu, C.-J.; Yang, T. Local existence with physical vacuum boundary condition to Euler equations with damping. J. Differential Equations 210 (2005), no. 1, 217-231.

[18] Yang, T. Singular behavior of vacuum states for compressible fluids. J. Comput. Appl. Math. 190 (2006), no. 1-2, 211-231.

DANIEL COUTAND

Heriot-Watt University

CANPDE

Maxwell Institute

for Mathematical Sciences

and

Department of Mathematics

Edinburgh EH14 4AS, UK

E-mail: D. Coutandehw.ac.uk

Received March 2010.

Revised May 2010.
STEVE SHKOLLER

University of California, Davis

Department of Mathematics

One Shields Avenue

Davis, CA 95616

E-mail: shkollere 